\title{
Nondissipative and Dissipative Momentum Deposition by Mountain Wave Events in Sheared Environments
}

\author{
CHRISTOPHER G. KRUSE AND RONALD B. SMITH \\ Yale University, New Haven, Connecticut
}

(Manuscript received 20 November 2017, in final form 18 April 2018)

\begin{abstract}
Mountain waves (MWs) are generated during episodic cross-barrier flow over broad-spectrum terrain. However, most MW drag parameterizations neglect transient, broad-spectrum dynamics. Here, the influences of these dynamics on both nondissipative and dissipative momentum deposition by MW events are quantified in a 2D, horizontally periodic idealized framework. The influences of the MW spectrum, vertical wind shear, and forcing duration are investigated. MW events are studied using three numerical models- the nonlinear, transient WRF Model; a linear, quasi-transient Fourier-ray model; and an optimally tuned Lindzen-type saturation parameterization-allowing quantification of total, nondissipative, and dissipative MW-induced decelerations, respectively. Additionally, a pseudomomentum diagnostic is used to estimate nondissipative decelerations within the WRF solutions. For broad-spectrum MWs, vertical dispersion controls spectrum evolution aloft. Short MWs propagate upward quickly and break first at the highest altitudes. Subsequently, the arrival of additional longer MWs allows breaking at lower altitudes because of their greater contribution to $u$ variance. As a result, minimum breaking levels descend with time and event duration. In zero- and positive-shear environments, this descent is not smooth but proceeds downward in steps as a result of vertically recurring steepening levels. Nondissipative decelerations are nonnegligible and influence an MW's approach to breaking, but breaking and dissipative decelerations quickly develop and dominate the subsequent evolution. Comparison of the three model solutions suggests that the conventional instant propagation and monochromatic parameterization assumptions lead to too much MW drag at too low an altitude.
\end{abstract}

\section{Introduction}

It is well known that gravity waves (GWs) flux horizontal momentum vertically in Earth's atmosphere, depositing momentum wherever they attenuate (e.g., Bretherton 1969; McLandress 1998; Alexander et al. 2010). Mountain waves (MWs), GWs generated by flow over mountains, attain this momentum flux (MF) through a pressure drag interaction with the mountains that generate them (Miles 1969; Smith 1979). That is, as the atmosphere flows over mountains, a pressure drag is exerted on the mountains by the atmosphere and momentum is transferred to Earth. An equal and opposite force is exerted by the mountain onto the lowest layers of the atmosphere, and MWs propagate this negative MF upward, depositing it wherever they dissipate. As much of Earth's terrain and resulting MW spectra are unresolved in general circulation models

Corresponding author: Christopher G. Kruse, christopher.kruse@ yale.edu and many weather prediction models, MW generation, propagation, and dissipation are parameterized. Including these parameterizations improves simulations of the atmospheric general circulation at nearly all levels (e.g., Holton 1983; Garcia and Solomon 1985; McLandress 1998; Palmer et al. 1986; McFarlane 1987), demonstrating the importance of MW momentum deposition. This deposition is important regionally as well (e.g., Lott and Miller 1997; Chen et al. 2007).

Conventionally, MW parameterizations make use of steady, linear theory, where the ambient environment is horizontally homogeneous and both the environment and the waves within it are steady. Steady theory considers forcing (i.e., cross-barrier flow) durations longer than the vertical propagation time of all MW scales, resulting in their presence at all altitudes (if not completely dissipated). Parameterizations apply steady theory at every instant, essentially assuming MWs have infinite vertical group velocity $c_{g z}$. In such a steady case, nondissipating linear MWs do not deposit momentum $\left(\partial_{z} \mathrm{MF}=0\right)($ Eliassen and Palm 1960) and, hence, do not 
influence their ambient flow. However, when the ambient environment is not horizontally homogeneous, wave refraction can result in persistent, cumulative nondissipative forcing to the ambient flow (Bühler and McIntyre 2003, 2005; Hasha et al. 2008), though, this situation is not considered here.

In reality, MW forcing is transient, allowing MWs to influence their ambient flow both nondissipatively and dissipatively. For example, when forcing is increased from zero, MWs are initially present near the terrain but are not aloft, producing nondissipative momentum deposition (i.e., $\partial_{z} \mathrm{MF} \neq 0$ ) that influences the ambient flow. When forcing ends, momentum deposition of the opposite sign occurs, which can return the ambient flow back to the initial profile. This nondissipative MW-ambient flow interaction has been termed self-acceleration (Fritts and Dunkerton 1984), direct GW-ambient flow coupling (Bölöni et al. 2016), and reversible. While nondissipative interactions are also often reversible, such interactions are referred to as nondissipative here. When MWs break or dissipate, permanent momentum deposition results.

Much of the previous work on nondissipative GWambient flow interactions has been highly idealized, considering a $2 \mathrm{D}$, horizontally periodic domain and horizontally monochromatic GWs with a specified vertically localized amplitude (e.g., Fritts and Dunkerton 1984; Dosser and Sutherland 2011; Fritts et al. 2015; Bölöni et al. 2016). In these studies, the prescribed vertical localization results in nondissipative momentum deposition and decelerations that can affect subsequent wave packet evolution and stability. Only recently has work been done on GW packets that are both vertically and horizontally localized (van den Bremer and Sutherland 2014). In all of this previous work, only quasi-monochromatic, analytically initialized GWs were considered. In reality, terrain and MW spectra are broad (Smith and Kruse 2017), having implications for MW amplitude and breaking levels as will be shown here.

Previous work is built upon here by considering broadspectrum, transient MWs generated by finite-duration flow over a single horizontally compact cosine mountain. There are two primary motivations for this added complexity. The first is that this complexity is more realistic, as MWs are actually generated by broad-spectrum terrain and forced by finite-duration flow. The second is that many MW drag (MWD) parameterizations in use neglect these complexities, characterizing subgrid-scale propagating MWs with a single wave of some horizontal wavelength that propagates upward instantly (e.g., Palmer et al. 1986; McFarlane 1987; Miller et al. 1989; Lott and Miller 1997; Kim and Arakawa 1995; Kim and Doyle 2005; Gregory et al. 1998; Webster et al. 2003). While significant improvements have been made to
MWD parameterizations since Lindzen (1981), the focus has been on nonlinearity in MW generation (i.e., flow blocking) and terrain anisotropy (Lott and Miller 1997; Webster et al. 2003; Kim and Doyle 2005; Smith and Kruse 2018) rather than on wave propagation, dissipation, and momentum deposition.

Transient, broad-spectrum MW events are studied here using three numerical models: the fully nonlinear, transient Weather Research and Forecasting (WRF) Model (Skamarock et al. 2008); the linear, quasi-transient Fourier-ray (FR) model (Broutman et al. 2002, 2006); and a Lindzen-type saturation parameterization (LSP; Lindzen 1981; McFarlane 1987) model. Key idealizations are the use of periodic lateral boundary conditions, 2D flow, and no planetary vorticity $(f=0)$. These idealizations limit the ambient flow response to MWD to deceleration, preventing MWD from being balanced by a pressure gradient or Coriolis force in a barrier jet-like response. The total (nondissipative plus dissipative) MW momentum deposition and ambient flow decelerations are trivially diagnosed in the MW-ambient flow coupled WRF solutions, as the model numerics time integrate the total momentum deposition and decelerate the flow from an initial profile. A pseudomomentum diagnostic is used to estimate nondissipative decelerations within these solutions. The linear FR model is also used to estimate linear nondissipative decelerations and to understand how finite forcing duration influences the evolution of a spectrum of MWs with a spectrum of vertical group velocities. Finally, dissipative decelerations by the LSP model are quantitatively evaluated against those in the WRF solutions.

\section{Background theory}

\section{a. The ambient horizontal momentum equation}

In the inviscid $f=0$ idealized flows considered here, the 2D flux-form $x$-momentum equation has the following simple form:

$$
\frac{\partial(\rho u)}{\partial t}+\frac{\partial}{\partial x}\left(\rho u^{2}+p\right)=-\frac{\partial(\rho u w)}{\partial z} .
$$

In a horizontally periodic domain, applying a horizontal domain average $\left[\overline{(\cdot)}=2 L^{-1} \int_{-L}^{L}(\cdot) d x\right.$ where $L$ is the domain half-width] to Eq. (1) removes the $\partial_{x}$ terms:

$$
\frac{\partial \overline{\rho u}}{\partial t}=-\frac{\partial \overline{\rho u w}}{\partial z} .
$$

Partitioning variables into an ambient (i.e., horizontally averaged) and perturbation part (e.g., $u=\bar{u}+u^{\prime}=U+u^{\prime}$ ) and neglecting density perturbations (Achatz et al. 2010), 
the ambient $x$-momentum equation takes the following simple form:

$$
\frac{\partial U}{\partial t}=-\frac{1}{\bar{\rho}} \frac{\partial\left(\bar{\rho} \overline{u^{\prime} w^{\prime}}\right)}{\partial z} .
$$

The right-hand side of Eq. (3) is conventionally defined as gravity wave drag (GWD) per unit mass, where GWD results from $\mathrm{GW}$ - or $\mathrm{MW}$-induced vertical gradients of $\mathrm{MF}=\bar{\rho} \overline{u^{\prime} w^{\prime}}$, the vertical flux of horizontal momentum. Hereafter, GWD by MWs is referred to as MWD. In this idealized setup, the ambient horizontal wind tendency is simply given by MWD, and the total ambient flow deceleration is the time-integrated MWD:

$\Delta U(z, t)=\int_{0}^{t}\left[-\frac{1}{\bar{\rho}(z)} \frac{\partial \mathrm{MF}\left(z, t^{\prime}\right)}{\partial z}\right] d t^{\prime}=\int_{0}^{t} \operatorname{MWD}\left(z, t^{\prime}\right) d t^{\prime}$.

Note that this domain-averaged $x$-mean momentum equation applies even when the terrain and MWD are horizontally localized. Localized MWD is quickly communicated horizontally throughout the domain in the form of a broad adverse pressure gradient, resulting in rather uniform deceleration (see section 6a) consistent with Achatz et al. (2010) and Rieper et al. (2013). Hence, $\Delta U$ and other domain-averaged diagnostics primarily presented in this paper are appropriate even for horizontally localized MWs.

\section{b. Nondissipative and dissipative $\Delta U$}

Both nondissipative and dissipative vertical gradients in MF (i.e., MWD) can result when MW forcing is transient. The former occurs as an MW propagates into some layer. Vertical gradients in MW amplitude result in nondissipative vertical gradients in MF and MWD, which alter the ambient flow as the MW propagates into a layer. MW dissipation also causes MWD; hence, nondissipative and dissipative MWD and decelerations are difficult to distinguish in Eq. (4).

The nondissipative and dissipative MWD and decelerations are perhaps most clearly seen in the generalized Lagrangian mean (GLM) equations. For example, the exact GLM $x$-momentum equation in the $2 \mathrm{D}$, horizontally periodic setup considered here is

$$
\frac{\partial U^{L}}{\partial t}=\frac{\partial P}{\partial t}+F,
$$

where $U^{L}=\bar{u}^{L}=\overline{u(\mathbf{x}+\boldsymbol{\xi}, t)}$ is the Lagrangian-mean zonal wind, $\boldsymbol{\xi}(x, t)=\xi(x, t) \hat{\mathbf{i}}+\eta(x, t) \hat{\mathbf{k}}$ is a $2 \mathrm{D}$ vector of wave-induced displacements, $\overline{(\cdot)}$ is the Eulerian horizontal average defined as before, $P=\overline{u^{l} \partial_{x} \xi}+\overline{w^{l} \partial_{x} \eta}$ is the $x$ pseudomentum per unit mass, $u^{l}$ and $w^{l}$ are Lagrangian perturbation velocities [e.g., $u^{l}=u(\mathbf{x}+\boldsymbol{\xi}, t)-\bar{u}^{L}$ ], and $F$ is a term that depends on Lagrangian perturbation body forces (e.g., viscosity) and diabatic heating (for a complete description, see Andrews and Mcintyre 1978; Bühler 2009). The $x$ pseudomomentum is a wave quantity that quantifies nondissipative wave forcing of the Lagrangian-mean flow. The last term in Eq. (5) is nonzero when waves are being generated or dissipated. In the present study, this term quantifies the dissipative forcing of the Lagrangian-mean flow. Equation (5) is valid for waves of finite amplitude.

For small-amplitude, slowly-varying waves in an environment with background shear, $P$ is given by $P=\bar{u}^{S}+\tilde{P}$, where $\bar{\varphi}^{S}=\bar{\varphi}^{L}-\bar{\varphi}=\overline{\boldsymbol{\xi} \cdot \nabla \varphi^{\prime}}+0.5 \overline{\eta^{2}} \partial_{z z} \bar{\varphi}$ is the Stokes correction for scalar $\varphi$, and $\tilde{P}$ is the Eulerian $x$ pseudomomentum per unit mass and is given by

$$
\tilde{P}=-\overline{\zeta^{\prime} \eta}-\frac{1}{2} \overline{\eta^{2}} \frac{\partial^{2} U}{\partial z^{2}}
$$

where $\zeta^{\prime}=\partial_{z} u-\partial_{x} w$ is the $y$ vorticity [Eq. (11.26) of Bühler 2009]. Substitution of $P=\bar{u}^{S}+\tilde{P}$ into Eq. (5) gives the Eulerian-mean flow tendency: $\partial_{t} U=\partial_{t} \tilde{P}+F$. Integrating this equation in time gives

$$
\Delta U=\Delta U_{\mathrm{nd}}+\Delta U_{d},
$$

where $\Delta U_{\text {nd }}=\tilde{P}$ (assuming zero initial $\tilde{P}$ ) is the nondissipative and $\Delta U_{d}=\int_{0}^{t} F \partial t$ the dissipative wave-induced ambient flow deceleration. Hence, as previously stated, when MWs are transient, they can induce both nondissipative and dissipative ambient flow decelerations.

To demonstrate how transience can influence the ambient flow physically, a relation between $\Delta U_{\text {nd }}$ and the MF spectrum of small-amplitude MWs is derived. First, variables [e.g., $\left.u^{\prime}(x)\right]$ periodic on $x \in[-L, L]$ are decomposed into wave components via Fourier series: $\hat{u}_{k_{n}}=\int_{-L}^{L} u^{\prime}(x) e^{-i k_{n} x} d x$, where $k_{n}=n \delta k$ is the angular wavenumber and $\delta k=2 \pi / 2 L=\pi / L$ is the fundamental wavenumber of the periodic domain. Parseval's theorem relates MF to its spectrum:

$$
\begin{aligned}
\operatorname{MF}(z) & =\frac{1}{2 \pi} \sum_{n=-\infty}^{\infty} \frac{\bar{\rho}(z)}{2 L} \operatorname{Re}\left[\hat{u}_{k_{n}}(z) \hat{w}_{k_{n}}^{*}(z)\right] \delta k \\
& =\sum_{n=-\infty}^{\infty} \widehat{\operatorname{MF}}_{k_{n}} \delta k,
\end{aligned}
$$

where the asterisk denotes complex conjugation.

Substituting Eq. (8) into Eq. (4) gives

$$
\Delta U(z, t)=-\sum_{-\infty}^{\infty} \frac{1}{\bar{\rho}} \int_{0}^{t} \frac{\partial \widehat{\mathrm{MF}}_{k_{n}}}{\partial z} d t^{\prime} \delta k .
$$


For small-amplitude, slowly-varying transient MWs in a slowly varying environment, $\widehat{\mathrm{MF}}_{k_{n}}$ is equivalent to the vertical flux of $x$-pseudomomentum density, $\widehat{\mathrm{PF}}_{k_{n}}$ (e.g., Scinocca and Shepherd 1992). The domain-averaged $x$-pseudomomentum density, $\bar{\rho} \hat{P}_{k_{n}}$, satisfies the conservation equation $\partial_{t}\left(\bar{\rho} \hat{P}_{k_{n}}\right)=-\partial_{z} \widehat{\mathrm{PF}}_{k_{n}}$ when waves are not dissipating, where $\widehat{\mathrm{PF}}_{k_{n}}=c_{g z} \bar{\rho} \hat{P}_{k_{n}}$ is the domain-averaged vertical flux of $x$-pseudomomentum density at $c_{g z}$. Substituting $\partial_{t}\left(\bar{\rho} \hat{P}_{k_{n}}\right)$ for $-\partial_{z} \widehat{\mathrm{MF}}_{k_{n}}$ in Eq. (9) gives

$$
\Delta U_{\mathrm{nd}}(z, t)=\sum_{-\infty}^{\infty} \hat{P}_{k_{n}} \delta k=\sum_{-\infty}^{\infty} \frac{1}{\bar{\rho}} \frac{\widehat{\mathrm{MF}}_{k_{n}}(z, t)}{c_{g z}(k, z)} \delta k,
$$

assuming zero initial pseudomomentum. The last equality follows either by noting $\hat{P}_{k_{n}}=\widehat{\mathrm{PF}}_{k_{n}} /\left(\bar{\rho} c_{g z}\right)=\widehat{\mathrm{MF}}_{k_{n}} /\left(\bar{\rho} c_{g z}\right)$ or from Eq. (6) neglecting the $\partial_{z z} U$ term and using WKB dispersion and polarization relations. Note that in a steady situation, $\widehat{\mathrm{MF}}_{k_{n}}$ is constant with height for small-amplitude MWs even in a sheared environment (Eliassen and Palm 1960). Given the dependence of $c_{g z}$ on $U(z), \Delta U_{\text {nd }}$ is dependent on the ambient shear profile in addition to the ambient density profile.

Consider a horizontally monochromatic wave packet with a vertically localized MF profile that is constant over some depth with a smooth ramp to zero above and below this constant value. This MF profile translates upward as the packet propagates vertically. Physically, Eq. (10) represents the time-integrated MWD at some level as a result of the passage of the leading and trailing edges of the packet through that level. The time it takes for the leading edge of the packet to pass through some level and, hence, the cumulative $\Delta U_{\mathrm{nd}}$, is dependent upon $c_{g z}$. Faster waves contribute less to $\Delta U_{\text {nd }}$ than slower waves.

Note that Eq. (10) implicitly defines a $\Delta U_{\text {nd }}$ spectrum. As noted by Smith and Kruse (2017), the MF spectrum is proportional to the terrain spectrum weighted by $|k|$ for linear, hydrostatic MWs. The hydrostatic $c_{g z}$ is also proportional to $k\left(\right.$ i.e., $\left.k U^{2} / N\right)$, implying the $\Delta U_{\text {nd }}$ spectrum is proportional to the terrain spectrum with no $k$ weighting. The largest contribution to $\Delta U_{\text {nd }}$ is made by the dominant terrain scales, which are typically the longest scales. The $u^{\prime}$ power spectrum is also proportional to the terrain spectrum (Smith and Kruse 2017), and so the longest scales dominate both the $\Delta U_{\text {nd }}$ and $u^{\prime}$ spectra.

The potential dissipative decelerations $\left(\Delta U_{d}\right)$ depend on the event duration. This follows from the $x$-impulse budget, found by multiplying Eq. (4) by $2 L \bar{\rho}(z)$ and integrating over depth $z_{d}$ :

$$
J_{0}=J_{\mathrm{MWD}}+J_{z_{d}} \cdot
$$

Here, $J_{0}=2 L \int_{0}^{\infty} \mathrm{MF}_{0} d t$ is the mountain impulse, where $\mathrm{MF}_{0}=-(2 L)^{-1} \int_{-L}^{L} p_{0} \partial_{x} h d x$ is the negative pressure drag, $J_{\mathrm{MWD}}=2 L \int_{0}^{z_{d}} \bar{\rho} \Delta U d z$ is impulse imparted onto the flow by MWD below $z_{d}$, and $J_{z_{d}}=2 L \int_{0}^{\infty} \operatorname{MF}\left(z_{d}\right) d t$ is the impulse transmitted through depth $z_{d}$. If MWs are completely dissipated below $z_{d}$, then all of the mountain impulse is exerted on the flow below $z_{d}$ (i.e., $J_{\mathrm{MWD}}=J_{0}$ ). In terms of the forcing-duration-averaged pressure drag $\left(\overline{\mathrm{MF}}_{0}^{\tau}\right)$, the mountain impulse is proportional to event duration $\left(J_{0}=2 L \overline{\mathrm{MF}}_{0}^{\tau} \tau\right)$, as are the potential dissipative decelerations $\left(\int_{0}^{z_{d}} \bar{\rho} \Delta U_{d} d z=\overline{\mathrm{MF}}_{0}^{\tau} \tau\right)$.

A fundamental difference between $\Delta U_{\text {nd }}$ and $\Delta U_{d}$ is that $\Delta U_{\text {nd }}$ reaches a maximum value when the maximum wave amplitude arrives at a particular level. However, if wave breaking occurs, then the potential $\Delta U_{d}$ is proportional to the event duration.

\section{Idealized case setup}

\section{a. Terrain}

Here, MWs are generated by flow over a simple compact cosine terrain defined by

$$
h(x)=\left\{\begin{array}{rr}
\frac{h_{m}}{2}\left[1+\cos \left(k_{h} x\right)\right], & |x| \leq d \\
0, & |x|>d
\end{array}\right.
$$

where $h_{m}$ is the maximum terrain height, $k_{h}=\pi / d$, and $d=100 \mathrm{~km}$ is the half-width of the terrain. The spectrum of this terrain (Fig. 1a) is narrow relative to real terrains, but it is still referred to as "broad," as a spectrum of scales is required to represent this terrain and the MWs it forces. Only two maximum terrain heights are used in this study: $h_{m}=50 \mathrm{~m}$ to produce nearly linear MWs through the 100-km-deep domain and $h_{m}=500 \mathrm{~m}$ to linearly launch MWs that then grow in amplitude with decreasing density aloft and break within the domain.

\section{b. Ambient stratification, wind profiles}

In all cases, constant stratospheric stratification $\left(N=\sqrt{g \partial_{z} \ln \theta}=0.02 \mathrm{~s}^{-1}\right)$ was specified. By also specifying surface pressure, $p_{0}=1000 \mathrm{hPa}$, temperature $T_{0}=288 \mathrm{~K}$, and assuming hydrostatic balance, $\bar{T}, \bar{p}$, and $\bar{\rho}$ profiles were found iteratively.

Ambient wind profiles are defined by

$$
\begin{aligned}
U_{t_{1}}(z)= & U_{z_{\infty}}\left[z+Z_{1} e^{-\left(z / Z_{1}\right)}-Z_{1}\right]-\frac{\Delta U}{2} \\
& \times\left\{1+\tanh \left[\frac{\pi}{2} \frac{\left(z-z_{i}\right)}{Z_{2}}\right]\right\}+U_{0} .
\end{aligned}
$$

The first term introduces positive shear. It produces a profile with zero shear at the surface but with speed 


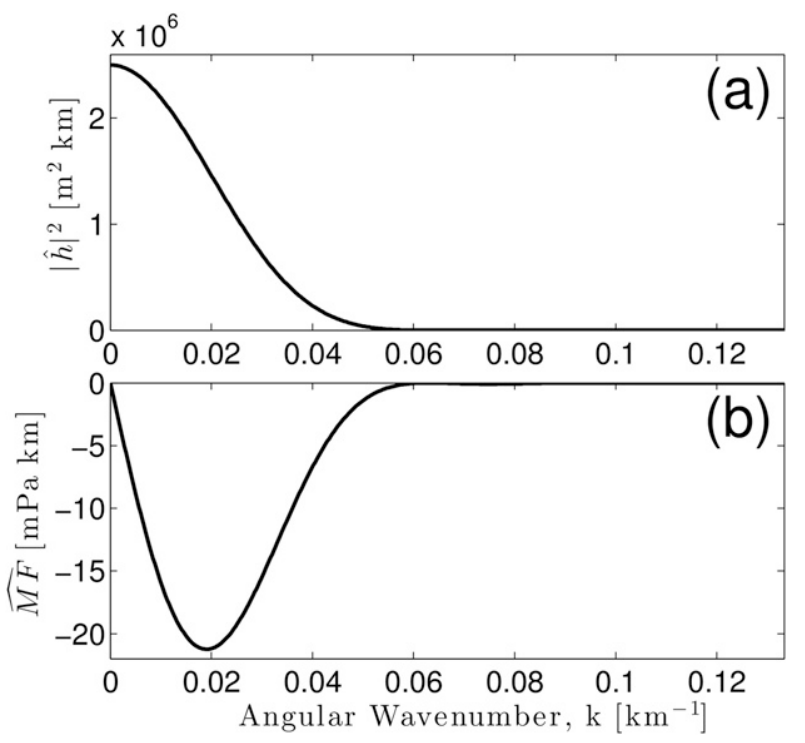

FIG. 1. (a) The terrain spectrum and (b) corresponding MF spectrum from nonhydrostatic linear theory [i.e., Eq. (22)] for terrain defined by Eq. (12). The spectra are plotted for wavenumbers from 0 to one-fifth the surface buoyancy cutoff $\left(0.2 k_{c}=0.2 \mathrm{~N} / U\right)$. The 2 -km-resolution terrain was zero padded by 9500 points on both sides to increase the spectral resolution in this figure.

and shear increasing with altitude, exponentially approaching a constant $U_{z \infty}$. The second term introduces negative shear and is used to produce wind profiles with a lower-stratospheric wind minimum. Finally, $U_{0}$ is the wind at the surface. In all cases considered, $Z_{1}=20 \mathrm{~km}$, $Z_{2}=8 \mathrm{~km}, z_{i}=15 \mathrm{~km}$, and $U_{0}=30 \mathrm{~m} \mathrm{~s}^{-1}$. The noshear $\left(U_{z \infty}=0 \mathrm{~s}^{-1}, \Delta U=0 \mathrm{~m} \mathrm{~s}^{-1}\right.$; black $)$, positive-shear ( $U_{z_{\infty}}=0.001 \mathrm{~s}^{-1}, \Delta U=0 \mathrm{~m} \mathrm{~s}^{-1}$; blue), and negative-shear $\left(U_{z \infty}=0.001 \mathrm{~s}^{-1}, \Delta U=30 \mathrm{~m} \mathrm{~s}^{-1}\right.$; red) wind profiles considered in this study are shown in Fig. 2. The negative-shear profile was chosen to represent a wintertime midlatitude profile with a lower-stratospheric wind minimum or MW valve layer (e.g., Kruse et al. (2016)), while the positiveshear profile is representative of a more poleward location below the polar night jet.

\section{c. Event forcing}

MW forcing is episodic, having some finite duration. Here, MWs are forced by accelerating the lowest $\sim 7-\mathrm{km}$

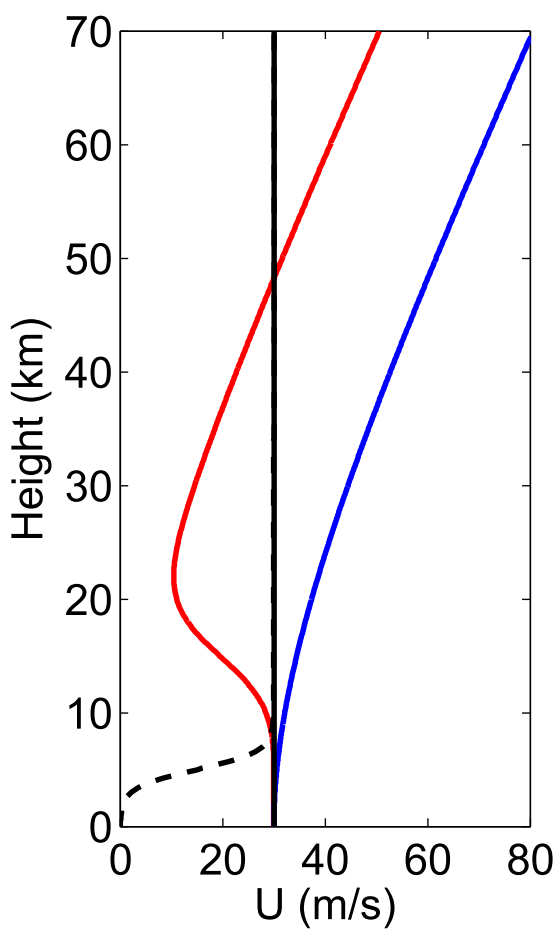

FIG. 2. No-shear (solid black, $U_{z \infty}=0 \mathrm{~s}^{-1}, \Delta U=0 \mathrm{~m} \mathrm{~s}^{-1}$ ), positive-shear (solid blue, $U_{z_{\infty}}=0.001 \mathrm{~s}^{-1}, \Delta U=0 \mathrm{~m} \mathrm{~s}^{-1}$ ), and negative-shear (solid red, $U_{z \infty}=0.001 \mathrm{~s}^{-1}, \Delta U=30 \mathrm{~m} \mathrm{~s}^{-1}$ ) ambient wind profiles computed via Eq. (13). In all cases, $U_{0}=30 \mathrm{~m} \mathrm{~s}^{-1}$. The dashed black curve approximately shows the initial wind profiles below $\sim 7 \mathrm{~km}$ in the WRF simulations in all cases.

flow from near zero to a profile defined by Eq. (13), allowing steady flow for some duration and decelerating the lowest flow back to the initial profile. The initial wind profile is given by

$$
U_{t_{0}}(z)=U_{t_{1}}\left\{0.5\left[\tanh \left(\frac{z-Z_{3}}{H_{d}}\right)+1\right]\right\}
$$

where $Z_{3}=5 \mathrm{~km}$ is the central height where the desired wind profile is transitioned nearly to zero below and $H_{d}=10 /(2 \pi) \mathrm{km}$ is a constant that controls the depth over which this transition occurs (e.g., Fig. 2, dashed). In the absence of any other forcing, the time-varying ambient flow is given by

$$
U(z, t)= \begin{cases}U_{t_{0}}(z)+0.5\left[U_{t_{1}}(z)-U_{t_{0}}(z)\right]\left[1-\cos \left(\pi t / \tau_{r}\right)\right], & 0 \leq t<\tau_{r} \\ U_{t_{1}}(z), & \tau_{r} \leq t \leq \tau_{r}+\tau_{\mathrm{nr}} \\ U_{t_{0}}(z)+0.5\left[U_{t_{1}}(z)-U_{t_{0}}(z)\right]\left(1+\cos \left\{\pi\left[t-\left(\tau_{r}+\tau_{\mathrm{nr}}\right)\right] / \tau_{r}\right\}\right), & \tau_{r}+\tau_{\mathrm{nr}}<t \leq \tau \\ U_{t_{0}}(z), & \text { else }\end{cases}
$$


where $\tau_{r}$ is the duration over which the lowest flow is ramped up or down $\left(\tau_{r}=20 \mathrm{~min}\right.$ in all experiments $), \tau_{\mathrm{nr}}$ is the duration of steady flow over the terrain (e.g., $23 \mathrm{~h}$ and $20 \mathrm{~min}$ ), and $\tau=2 \tau_{r}+\tau_{\mathrm{nr}}$ is the total forcing duration (e.g., 24h).

\section{Models}

\section{a. Nonlinear WRF Model}

The Weather Research and Forecasting (WRF, version 3.8.1) Model is used to simulate the fully nonlinear evolution and breakdown of MW events. The WRF Model is used in a $2 \mathrm{D}$, horizontally periodic, inviscid, zero planetary vorticity $(f=0)$, and dry idealized configuration. The WRF domain is $100 \mathrm{~km}$ deep and $1000 \mathrm{~km}$ wide (unless otherwise indicated), with terrain specified by Eq. (12). A horizontal (vertical) resolution of $2 \mathrm{~km}(500 \mathrm{~m})$ was used. In the linear $\left(h_{m}=50 \mathrm{~m}\right)$ and wave breaking $\left(h_{m}=500 \mathrm{~m}\right)$ cases, time steps of 10 and $5 \mathrm{~s}$ were used, respectively. A traditional Rayleigh friction sponge layer (namelist option "damp_opt $=2$ ") was specified in the upper $30 \mathrm{~km}$ of the domain to prevent artificial reflection.

Finite-duration cross-barrier flow was included by taking the time derivative of Eq. (15) and adding this tendency as a horizontally uniform forcing in WRF's $x$-momentum equation. In the absence of any other forcing, the WRF Model accurately reproduced the transient ambient flow defined by Eq. (15) (not shown).

The ambient flow responds to MWD by decelerating in the 2D, horizontally periodic setup [e.g., Eq. (4)]. This allows the total $\Delta U$ to be trivially diagnosed as deviations from Eq. (15), as the WRF Model numerics time integrate both nondissipative and dissipative MWD. Within the WRF solutions, $\Delta U_{\text {nd }}$ is estimated by the small-amplitude $x$-pseudomomentum relation [Eq. (6)] and $\Delta U_{d}$ is then estimated by $\Delta U-\Delta U_{\mathrm{nd}}$.

\section{b. Linear FR model}

In addition to estimating $\Delta U_{\text {nd }}$ via Eq. (6), a $2 \mathrm{D}$ version of the quasi-transient, linear FR model (Broutman et al. 2006 and references therein) is used to estimate and study the dynamics leading to linear nondissipative MW momentum deposition and $\Delta U_{\text {nd }}$. This model takes into account the full terrain spectrum, finite-duration forcing, and vertical dispersion. In short, this model computes a zero-phase-speed MW spectrum from the terrain spectrum, propagates this spectrum upward via the $c_{g z}$ spectrum, and uses pseudomomentum conservation to determine wave amplitudes, taking into account a slowly varying profile of wind and stability. MW dissipation is not represented, allowing linear estimates of nondissipative momentum deposition and flow decelerations.
Following Eckermann et al. (2015, 2016), the FR model makes use of the 2D GW dispersion relation,

$$
m(k, z)=-\operatorname{sgn}(\hat{\omega})\left[\frac{k^{2}\left(N^{2}-\hat{\omega}^{2}\right)}{\hat{\omega}^{2}}\right],
$$

where $\hat{\omega}=\hat{\omega}(k, z)=-U(z) k$ is the intrinsic frequency for stationary MWs. The $\operatorname{sgn}(\hat{\omega})$ term ensures an upward vertical group velocity, which is given by

$$
c_{g z}(k, z)=-\frac{m \hat{\omega}}{k^{2}+m^{2}} .
$$

The FR solution for vertical displacement $\eta$ in Fourier space is

$$
\begin{aligned}
\hat{\eta}(k, z)= & {\left[\frac{\bar{\rho}(0)}{\bar{\rho}(z)}\right]^{1 / 2} \hat{h}(k)\left[\frac{c_{g z}(k, 0) \hat{\omega}(k, z)}{c_{g z}(k, z) \hat{\omega}(k, 0)}\right]^{1 / 2} } \\
& \times \exp \left[i \int_{0}^{z} m\left(k, z^{\prime}\right) d z^{\prime}\right]
\end{aligned}
$$

where the density term on the left accounts for amplitude growth with decreasing density, $\hat{h}(k)$ is the Fourier transform of the topography (Fig. 1a), and the bracketed term ensures pseudomomentum conservation and MF invariant with height. Fields in physical space are computed via the inverse Fourier transform,

$$
\eta(x, z, t)=\int_{-\infty}^{\infty} F_{\text {sfc }}\left[t-t_{\text {prop }}(k, z)\right] \hat{\eta}(k, z) e^{i k x} d k .
$$

Time dependence is introduced only through the surface forcing term,

$$
F_{\mathrm{sfc}}(t)=\frac{U(0, t)}{U_{0}},
$$

which is the ambient surface wind speed function [here defined by Eq. (15)] normalized by its maximum value, $U_{0}=30 \mathrm{~m} \mathrm{~s}^{-1}$, having values in the range of $F_{\text {sfc }} \in[0,1]$. Vertical dispersion (i.e., the $c_{g z}$ spectrum) is taken into account by shifting $F_{\text {sfc }}$ by $-t_{\text {prop }}(k, z)$, which is defined by

$$
t_{\text {prop }}(k, z)=\int_{0}^{z} \frac{d z^{\prime}}{c_{g z}\left(k, z^{\prime}\right)} \text {. }
$$

This shift gives every wave component a smooth vertical $\widehat{\mathrm{MF}}$ profile with the shape of the surface wind speed function stretched and translated vertically based on the component's vertical group velocity. When $F_{\text {sfc }}\left(t-t_{\text {prop }}\right)=1$, Eq. (19) gives the steady, linear wave solution, where all scales that can be are present at all altitudes.

The nondissipative ambient flow reductions were estimated within the FR solutions both by time-integrating 
MWD [Eq. (4)] and from the MF spectrum [Eq. (10)]. Both estimates agreed very well (not shown). Equation (10) was used to estimate $\Delta U_{\text {nd }}$ spectrally in the FR solutions.

There are a few important limitations to this version of the FR model. One is that most nonhydrostatic effects (i.e., reflection, evanescence tunneling) are neglected. However, the scales launched by the chosen terrain (Fig. 1) do not encounter reflection levels in any of the shear cases considered. Another important limitation is that only stationary waves are represented. This is unrealistic for MWs generated from transient forcing, as waves present in an accelerating (decelerating) atmosphere attain positive (negative) Earth-relative phase speeds (e.g., Chen et al. 2005). However, this is primarily an issue at the end of events and, overall, there is good quantitative agreement between the WRF and FR solutions (e.g., section 5). A final important limitation is that the MWs are not coupled to the ambient flow, so $\Delta U_{\text {nd }}$ cannot feed back onto the MWs and influence their evolution.

Originally, the FR model was configured on the 2-kmresolution, 1000-km-wide WRF domain. This resulted in poor spectral resolution, leading to discontinuous vertical profiles of domain-averaged quantities as the different scales propagate up at different speeds. To address this issue, the spectral resolution of the FR model was increased by using a $20000-\mathrm{km}$-wide domain. While domain-integrated MF spectra were nearly unaffected, the domain-averaged MF spectra were a factor of 20 smaller. FR computed MF and $\Delta U_{\text {nd }}$ were multiplied by 20 to produce MF and $\Delta U_{\text {nd }}$ comparable to those in the 1000-km-wide WRF solutions.

\section{c. MWD parameterization}

The MWD parameterization used here is a Lindzentype saturation parameterization (LSP model; e.g., Lindzen 1981) developed following McFarlane (1987) and Eckermann et al. (2015). It uses the standard parameterization assumptions: a monochromatic wave field; instant vertical propagation; waves saturate (i.e., not allowed to attain amplitude required to induce static instability or, equivalently, reverse the flow); $2 \mathrm{D}$ hydrostatic flow; and steady, horizontally uniform ambient environment. Its inputs are an ambient wind [e.g., Eq. (13)] and density profile, and a source-level momentum flux, $\mathrm{MF}_{0}$.

$\mathrm{MF}_{0}$ is computed from the terrain spectrum via linear, nonhydrostatic theory,

$$
\begin{aligned}
\mathrm{MF}_{0} & =\int_{-\infty}^{\infty} \frac{\bar{\rho}_{0} N U_{0}}{4 \pi L}\left[1-\frac{(U k)^{2}}{N^{2}}\right]^{1 / 2}|k||\hat{h}(k)|^{2} d k \\
& =\int_{-\infty}^{\infty} \widehat{\operatorname{MF}}_{0}(k) d k,
\end{aligned}
$$

in order to supply the parameterization with the most accurate $\mathrm{MF}_{0}$. The $\mathrm{MF}_{0}$ spectrum, $\widehat{\mathrm{MF}}_{0}$, is plotted in Fig. 1b. In the LSP solutions, the 20-min ramps up and down of the source-level winds are neglected, so $\mathrm{MF}_{0}$ is constant for the forcing duration. For the $h_{m}=500-\mathrm{m}$ hill, $\mathrm{MF}_{0}=-196.504 \mathrm{mPa}$. This value agreed well with the WRF solutions, implying the momentum fluxed out the bottom, and the potential impulses onto the ambient flows of the WRF and LSP domains were nearly the same.

The propagation part of the parameterization requires a choice of parameterized wavenumber $k_{p}$ chosen here to be that contributing the most to the MF spectrum (Fig. 1b). This choice gives a wavelength of $\lambda_{x}=328 \mathrm{~km}$. Given this large wavelength, hydrostatic linear theory is used to compute MW amplitudes given an ambient profile.

With $\mathrm{MF}_{0}$, a source-level $u^{\prime}$ amplitude $\check{u}$ is computed via

$$
\check{u}_{0}=\left(\frac{-2 N \mathrm{MF}_{0}}{\bar{\rho}_{0} U_{0} k_{p}}\right)^{1 / 2} .
$$

The wave amplitude is then computed at the next highest model level assuming MF conservation:

$$
\check{u}_{k+1}=\check{u}_{k}\left(\frac{U_{k}}{U_{k+1}} \frac{\bar{\rho}_{k}}{\bar{\rho}_{k+1}}\right)^{1 / 2} .
$$

When $\check{u}_{k+1} \leq U_{k+1}$, then $\check{u}_{k+1}$ is unaltered and the momentum flux is kept constant $\left(\mathrm{MF}_{k+1}=\mathrm{MF}_{k}\right)$. When $\check{u}_{k+1}>U_{k+1}$, then the wave amplitude is reduced to the saturation value, $\check{u}_{k+1}=U_{k+1}$, and the saturated MF at this level is computed via

$$
\mathrm{MF}_{k+1}=-\frac{\bar{\rho}_{k+1} k_{p} U_{k+1}^{3}}{2 N} .
$$

Iterating this method up all model levels produces an MF profile, from which $\mathrm{MWD}_{k+1}=-1 / \bar{\rho}_{k+1}\left(\mathrm{MF}_{k+1}-\right.$ $\left.\mathrm{MF}_{k}\right) / \Delta z$ is computed.

The 1000-km-wide domain-averaged $\mathrm{MF}_{0}$ could be provided to the LSP model to produce flow tendencies. However, this $\mathrm{MF}_{0}$ is effectively diluted because only $20 \%$ of the domain contains terrain, and waves with this $\mathrm{MF}_{0}$ would have unrealistically small amplitudes. To address this dilution, the $\mathrm{MF}_{0}$ is put into a single $200-\mathrm{km}$ wide "grid cell" containing the terrain by scaling $\mathrm{MF}_{0}$ by $2 L / 2 d=5$. The MWD parameterization is then run to provide an MF profile. This profile is then unscaled by multiplying by $2 d / 2 L=1 / 5$, which allows any resulting MWD to be applied uniformly to the $1000-\mathrm{km}$-wide domain containing 5 times the momentum of the grid cell.

After an MF profile is computed, a $10-\mathrm{km}$ vertical moving average smoother is applied to the profile. Without this smoothing, MWD is strongly dependent 

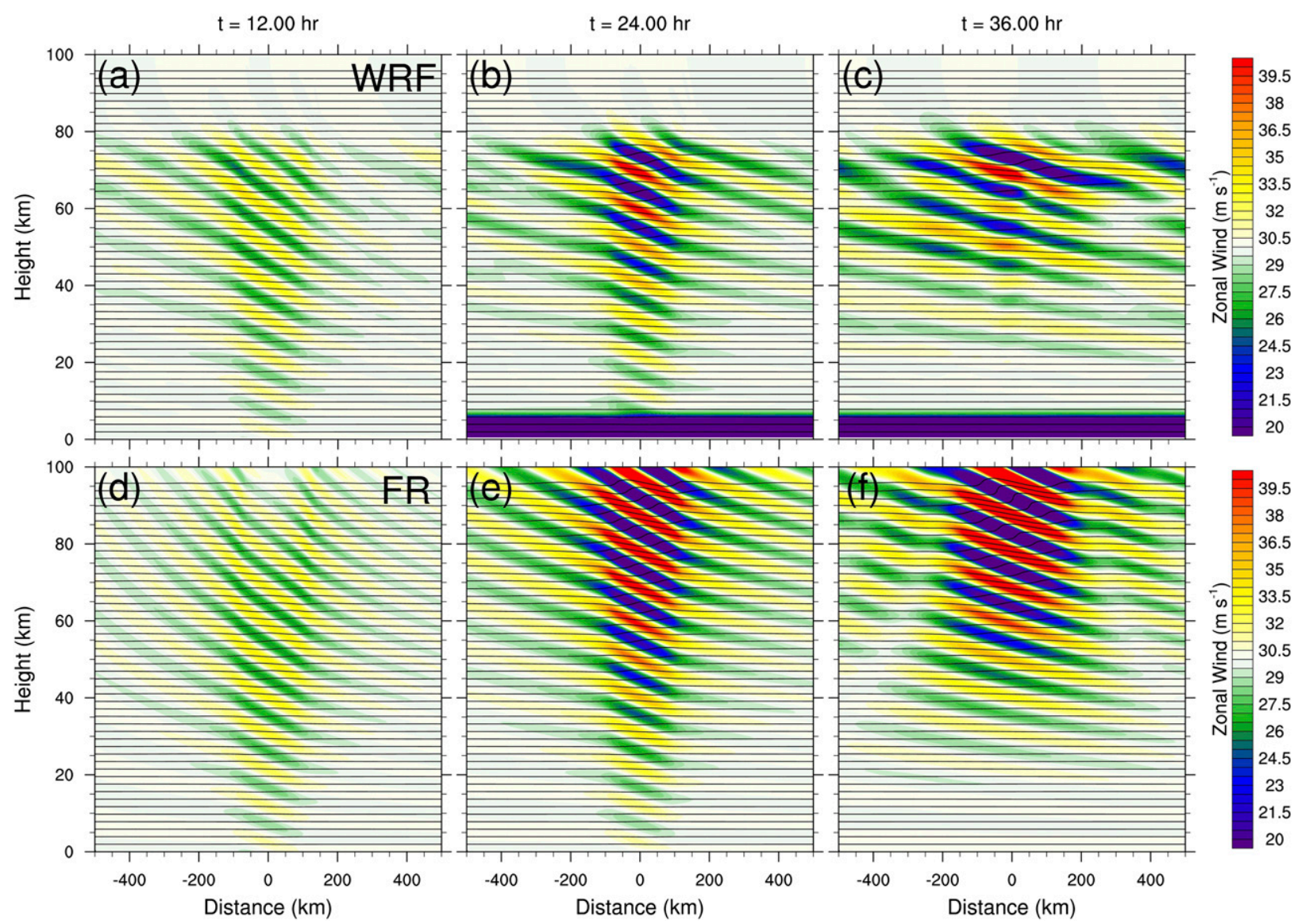

FIG. 3. Vertical sections from the no-shear case with small-amplitude terrain $\left(h_{m}=50 \mathrm{~m}\right)$ and a forcing duration of $24 \mathrm{~h}$. The full zonal wind $\left(\mathrm{m} \mathrm{s}^{-1}\right)$ is shown with color shading, and potential temperature (K) is contoured at $t=(\mathrm{a}),(\mathrm{d}) 12$, (b),(e) 24, and (c),(f) $36 \mathrm{~h}$. The damping layer in the WRF solutions starts at $z=70 \mathrm{~km}$.

on vertical resolution (not shown), as this resolution sets the smallest scale over which parameterized MWs could be dissipated. The smoother enforces a vertical dissipation scale independent of resolution comparable to the vertical wavelength in the no-shear case $\left(\lambda_{z}=2 \pi U / N=9.42 \mathrm{~km}\right)$.

This 1D LSP model was implemented on the same $\Delta z=500-\mathrm{m}$ vertical grid as the WRF and FR models. Flow tendencies from this parameterization were used to decelerate initial profiles [Eq. (13)] via forward finite difference with a time step of $\Delta t=5 \mathrm{~min}$. A range of time steps from 20 to 2 min were tested, and solutions were found to be insensitive to different time steps in this range, suggesting solution convergence.

\section{Nondissipative momentum deposition}

Here, a small-amplitude MW case is studied to quantify nondissipative momentum deposition and decelerations. MWs are forced by $\tau=24 \mathrm{~h}$ of flow (unless otherwise indicated) across a mountain of height $h_{m}=50 \mathrm{~m}$.

The WRF and FR solutions are compared in Figs. 3-6. In all of these comparisons, there is good quantitative agreement during the forcing period. Notable differences occur immediately after the forcing $(t=24 \mathrm{~h})$. Prior to the ramp down in the WRF solutions, an approximately steady MW field developed. As the lowest $\sim 5 \mathrm{~km}$ of the ambient flow are quickly and uniformly decelerated to rest, the previously steady MWs begin to travel upstream. These upstream and upwardpropagating MWs are not accounted for in the FR model and are apparently responsible for the MF maxima (Figs. 4, 5) and corresponding $\Delta U_{\text {nd }}$ differences (Fig. 6) right after the forcing duration. Despite these differences, there is good overall agreement between the two solutions, suggesting the FR model dynamics are correctly representing most of the wave dynamics in the WRF Model. The influences of this imposed deceleration are not further investigated here. 


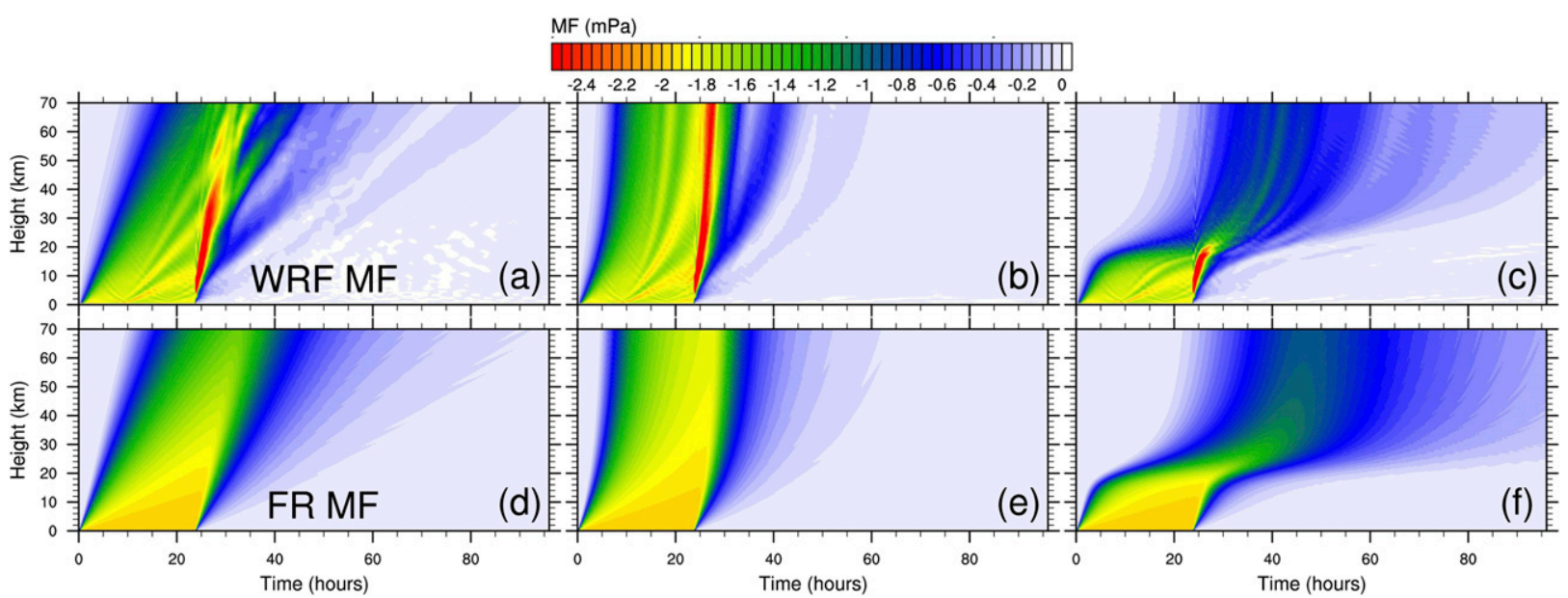

FIG. 4. Horizontal domain-averaged vertical flux of horizontal momentum ( $\left.\mathrm{MF}=\bar{\rho} \overline{u^{\prime} w^{\prime}}, \mathrm{mPa}\right)$ is shown as a function of height and time from the (a)-(c) WRF and (d)-(f) FR models for the short mountain $\left(h_{m}=50 \mathrm{~m}\right)$. Results are from the (left) no-shear, (center) positiveshear, and (right) negative-shear cases.

Initially, there are no waves and, hence, no MF (Fig. 4). After the ramp up of flow, a nondissipative vertical gradient in MF develops as a result of the presence of MWs near the topography with still none aloft. During the forcing period, the MF profile disperses vertically. After the forcing period, the MWs depart the lowest layers, reducing MF and reversing the sign of the nondissipative momentum deposition.

The MF profile evolution in the FR solutions, which well reproduces that in the WRF solutions, is controlled by the $c_{g z}$ spectrum. For the broad terrain considered, the hydrostatic $c_{g z}=k U^{2} / N$ is valid. The short-wave portion of the spectrum propagates upward quickly, while the long-wave portion propagates slowly. This spectrum controls how much of the source spectrum [e.g., Eq. (22); Fig. 1b] and MF can be present at a particular altitude and time. This is demonstrated in Fig. 5, where the $z=50-\mathrm{km}$ MF spectrum evolution is plotted in the WRF and FR solutions for forcing durations of $\tau=12,24$, and $36 \mathrm{~h}$. Initially, only the shortest scales are present as a result of the fast propagation. As time increases, the longer waves arrive and the spectrum aloft becomes increasingly complete. This is particularly clear in the $\tau=36$-h forcing duration case (Figs. 5c,g).

Note that during the forcing period, as long as the entire wave spectrum is not present, MF is not constant with height (i.e., nondissipative $\partial_{z} \mathrm{MF}>0$ ), as the MF spectrum is increasingly complete with decreasing altitude. MF is constant with height only where the spectrum is entirely present, which occurs only in the lowest layers where the slow, long MWs have had enough time to propagate. Otherwise, MF is, for the most part, not constant with height for transient, broad-spectrum MWs (Fig. 4).
After the event forcing, the longer waves are still arriving at $z=50 \mathrm{~km}$, acting to increase MF, while the shorter waves rapidly depart and decrease MF. The wave spectrum narrows and shifts to longer scales with time. The total MF decreases with time as a result (Figs. 5d,h).

The corresponding $\Delta U_{\text {nd }}$ are shown within the WRF and FR solutions in Fig. 6. Here, WRF $\Delta U_{\text {nd }}$ was computed via Eq. (6) using potential temperature perturbations to compute vertical displacements. The FR $\Delta U_{\text {nd }}$, computed via Eq. (10), gives a linear estimate of nondissipative decelerations without wave-ambient flow coupling, while WRF $\Delta U_{\text {nd }}$ gives an estimate of the total nondissipative decelerations with wave-ambient flow coupling, as WRF allows waves to influence the mean and these changes to subsequently influence MW propagation. Comparison of WRF $\Delta U$ with WRF $\Delta U_{\text {nd }}$ in Fig. 6 shows that in this short-mountain case, nondissipative decelerations dominate. The FR-predicted $\Delta U_{\text {nd }}$ agrees well with that in WRF, with differences resulting from a lack of wave-ambient flow coupling and accounting for only zero-phase-speed MWs in the FR model.

Some permanent dissipative decelerations are present in these WRF solutions, evident as horizontal streaks in the first and third rows of Fig. 6. While the mountain is small, the MWs become somewhat nonlinear in the noshear and negative-shear cases (i.e., $u^{\prime} / U$ exceed 0.4 , not shown) where dissipative decelerations initially appear. This layering is apparently due to enhanced momentum deposition in the vertically recurring and localized regions of steepening above the highest terrain by hydrostatic MWs launched by localized terrain (e.g., Smith 1977). Hydrostatic MWs launched by horizontally 


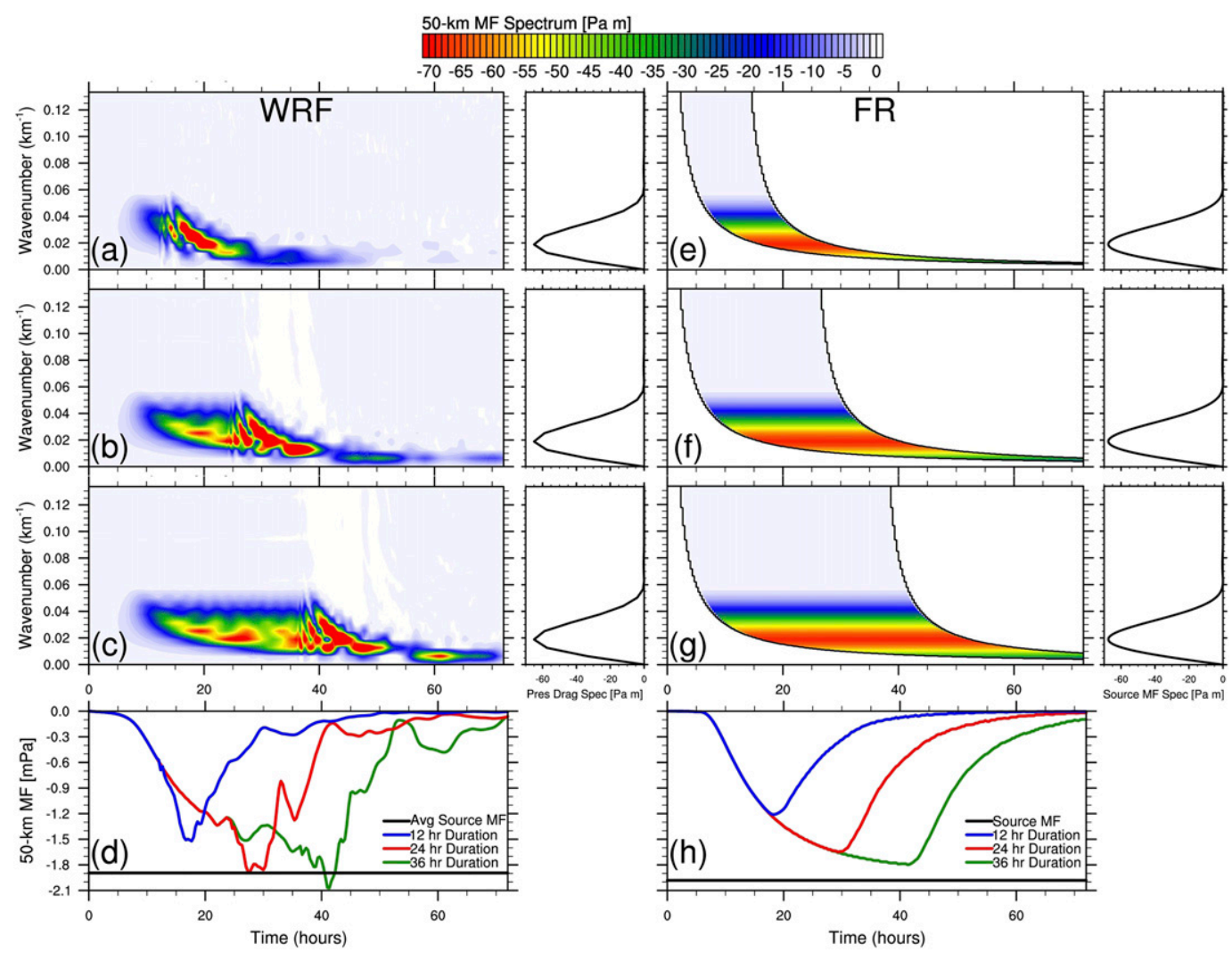

FIG. 5. MF spectrum evolution at $z=50 \mathrm{~km}$ in the (a)-(d) WRF and (e)-(h) FR solutions for forcing durations of (a),(e) 12, (b),(f) 24, and (c),(g) $36 \mathrm{~h}$ in the no-shear case. (d),(h) The corresponding MF time series for the three durations. The forcing-duration-averaged source spectra are shown on the rhs of (a)-(c) and (e)-(g), and the integral of these spectra gives the total source MF shown in black in (d) and (h).

localized terrain steepen periodically in the vertical, with the spacing between steepening levels given by the vertical wavelength $\left(\lambda_{z}=2 \pi U / N\right)$. In the no-shear case, $\lambda_{z}=9.42 \mathrm{~km}$. In the negative-shear case, $\lambda_{z}$ decreases from $9.42 \mathrm{~km}$ at the surface and to $\sim 3.3 \mathrm{~km}$ at $z=21 \mathrm{~km}$. The vertical spacing of the $\Delta U_{d}$ layers matches the predicted vertical wavelengths very well.

In the FR solutions, $\Delta U_{\text {nd }}$ is inversely proportional to $c_{g z}$ [Eq. (10)] and, hence, $U^{2}$ for all MW scales. In the positive-shear case, $\Delta U_{\text {nd }}$ is reduced relative to the noshear case (cf. Figs. 6d,j with Figs. 6e,k). The region of minimum wind speed near $z=21 \mathrm{~km}$ in the negativeshear case enhances $\Delta U_{\text {nd }}$ (cf. Figs. 6d,j with Figs. 6f,l).

The $c_{g z}$ spectrum controls how $\Delta U_{\text {nd }}$ evolves as well. While the wave spectrum narrows with time after the wave forcing, it is also increasingly shifted to longer scales. As noted in section $2 \mathrm{c}$, the $\Delta U_{\mathrm{nd}}$ spectrum is proportional to the terrain spectrum, which is dominated by the longest scales (Fig. 1a). The larger $\Delta U_{\text {nd }}$ contribution by long MWs somewhat compensates for the narrowing of the spectrum, resulting in a surprisingly slow return of the ambient flow to the initial profile aloft.
This is especially apparent in the no-shear and negativeshear cases, where the ambient flow above $z=50 \mathrm{~km}$ has not fully recovered to the initial profile by $72 \mathrm{~h}$ after the event forcing (Fig. 6). The slow arrival of the long-wave portion of the MW spectrum is supported by Deep Propagating Gravity Wave Experiment (DEEPWAVE) observations presented by Portele et al. (2018), where the appearance of mesospheric long-wave activity was delayed about a day from the period of maximum flow across New Zealand.

\section{Dissipative and nondissipative momentum deposition}

Here, the numerical experiments of the previous section are repeated with an $h_{m}=500$-m-high mountain. With $U_{0}=30 \mathrm{~m} \mathrm{~s}^{-1}$ and $N=0.02 \mathrm{~s}^{-1}$, the maximum source nonlinearity ratio is NLR $=u^{\prime} / U=N h / U=1 / 3$, but it still indicates that the low-level flow and wave generation is nearly linear. Nonlinearity and wave breaking develop aloft within the WRF solutions as the MW amplitude grows with decreasing density. 


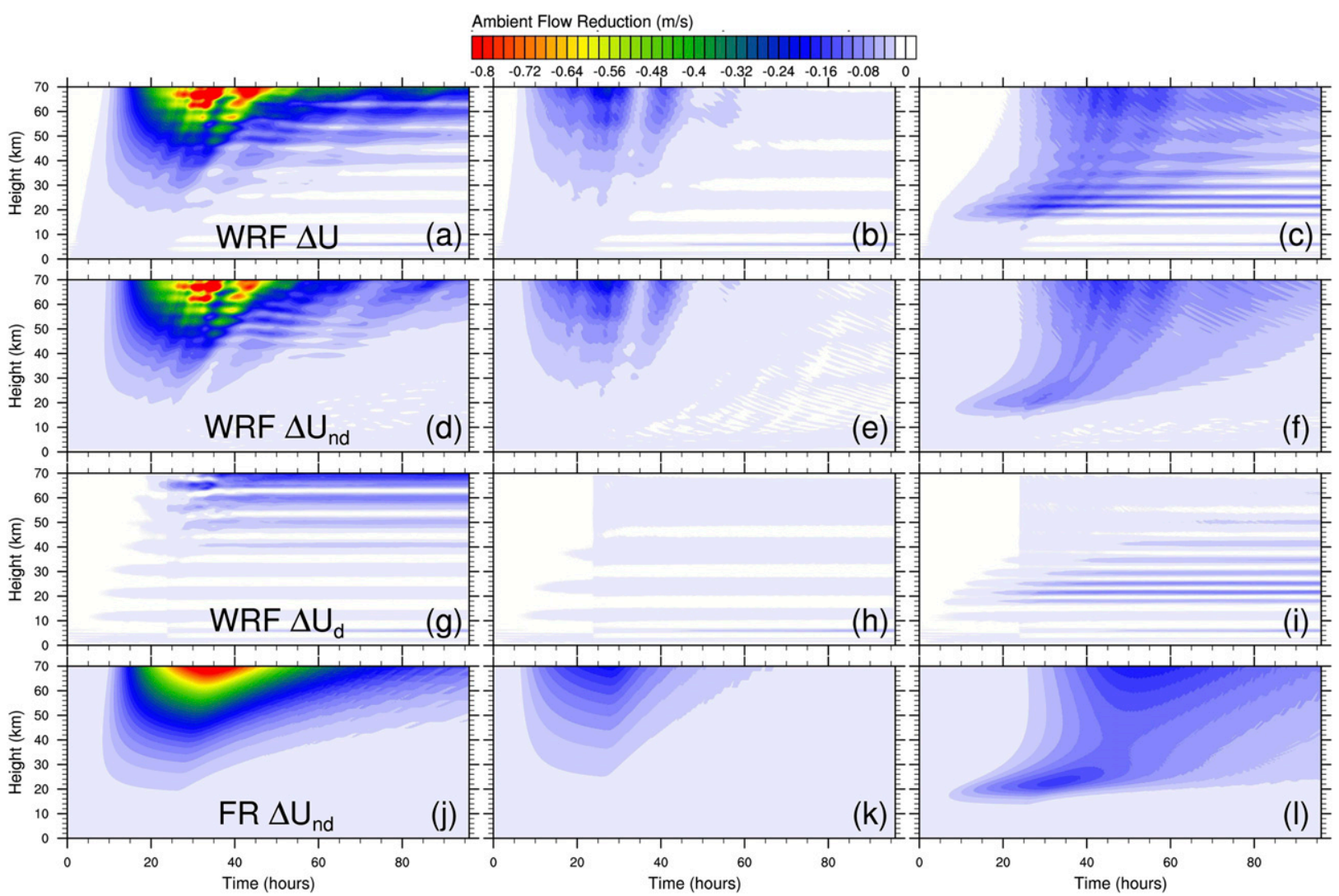

FIG. 6. Nondissipative and dissipative ambient flow decelerations $\left(\mathrm{m} \mathrm{s}^{-1}\right)$ are shown as a function of height and time from the (a)-(i) WRF and (j)-(1)FR models for the short mountain $\left(h_{m}=50 \mathrm{~m}\right)$. The total decelerations $\Delta U$, nondissipative decelerations $\Delta U_{\text {nd }}$ computed via Eq. (6), and dissipative decelerations $\left(\Delta U_{d}=\Delta U-\Delta U_{\mathrm{nd}}\right)$ are shown in the WRF solutions in (a)-(c), (d)-(f), and (g)-(i), respectively. Results are from the (left) no-shear, (center) positive-shear, and (right) negative-shear cases.

\section{a. Initial evolution}

Vertical sections within the WRF and FR solutions are shown in Fig. 7 for the no-shear case at $t=6,12$, and $18 \mathrm{~h}$. At $t=6 \mathrm{~h}$, there is good agreement between the WRF and FR solutions below $z=50 \mathrm{~km}$. Above, there are differences, possibly as a result of underestimated vertical extent of the MWs and/or the neglect of downstreampropagating MWs generated during the ramp up by the FR model. At $t=12 \mathrm{~h}$, the large amplitude waves aloft have just begun to break in the WRF solution, as evidenced by small-scale isentropic undulations near $z=$ $70 \mathrm{~km}$. Despite the nonlinearity, there is still decent agreement between the two solutions, suggesting the linear FR model is useful up to the point of wave breaking. By $t=18 \mathrm{~h}$, nonlinear dynamics in the WRF solutions have caused them to diverge from the FR solutions.

\section{b. Response to localized MWD}

Because the mountain is horizontally localized, so are the MWs and the MWD they exert on the flow. Given this localized forcing, how does the flow respond? Is the response also local? Regional? Domainwide? How do nondissipative and dissipative MWD and their responses compare? To answer these questions, a crude regional flow deceleration equation is derived by defining a smoothing operator, partitioning variables into a regional ambient and perturbation part, and applying this smoother to the $x$-momentum equation.

The smoothing operator is a $2 \mathrm{D}$ moving area average in the $x-z$ plane,

$$
\langle a\rangle(x, z)=\frac{1}{2 L_{x} 2 L_{z}} \int_{x-L_{x}}^{x+L_{x}} \int_{z-L_{z}}^{z+L_{z}} a(x, z) d x d z
$$

where $a(x, z)$ is some $2 \mathrm{D}$ variable, and $L_{x}=150 \mathrm{~km}$ and $L_{z}=5 \mathrm{~km}$ are the horizontal and vertical half-widths of the smoother, respectively. The width of the smoother is $100 \mathrm{~km}$ wider than the extent of the terrain, and the depth $\left(2 L_{z}=10 \mathrm{~km}\right)$ is about one vertical wavelength of a hydrostatic MW in the no-shear case $\left(\lambda_{z}=9.42 \mathrm{~km}\right)$. In this subsection only, variables are partitioned via $a=\langle a\rangle+a^{\prime}$, with the exception of $\rho$, where density perturbations are neglected [i.e., $\rho=\bar{\rho}(z)$ ]. Applying the 

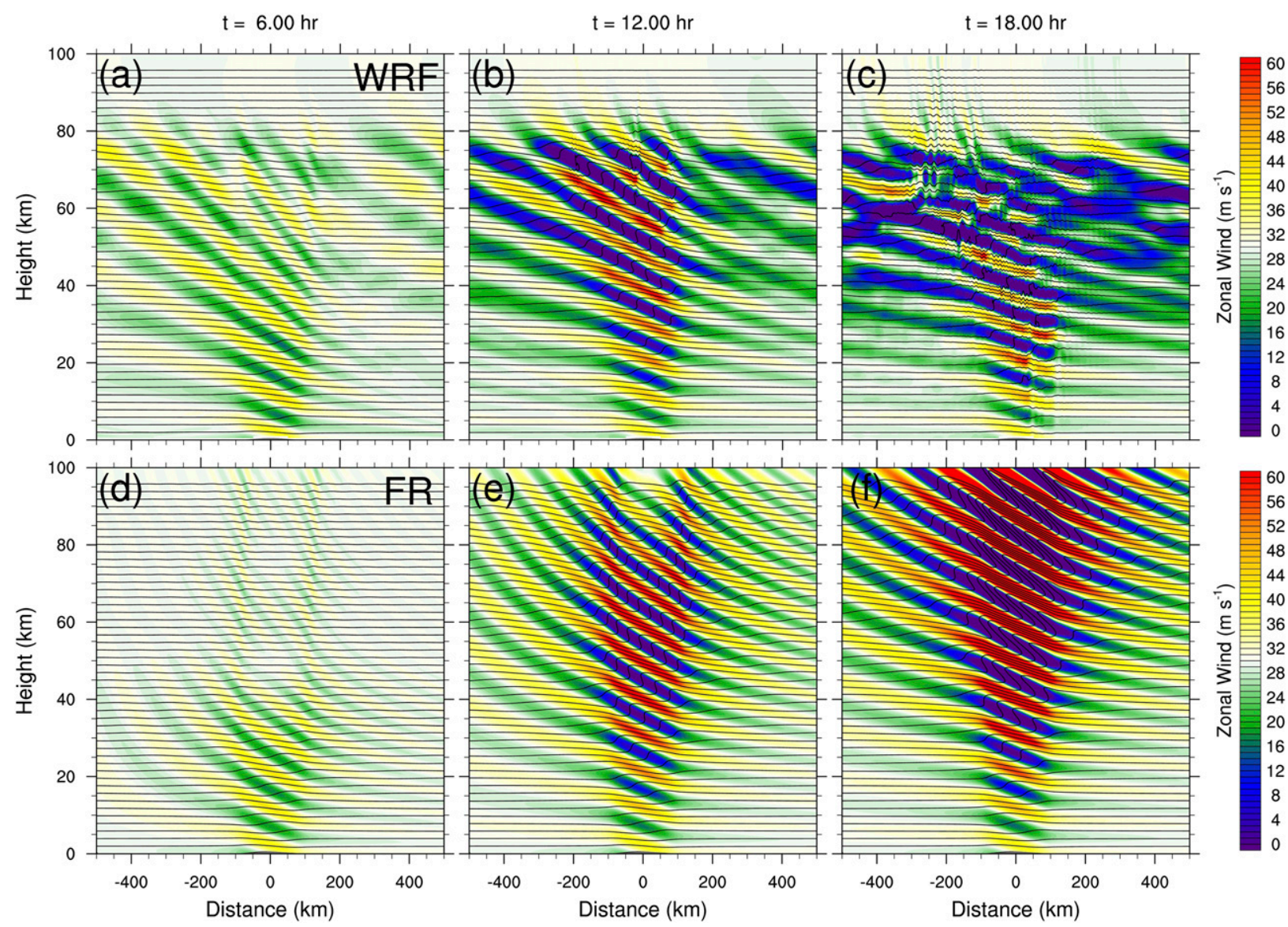

FIG. 7. As in Fig. 3, but for the tall mountain case $\left(h_{m}=50 \mathrm{~m}\right)$ with different valid times and color scale. Wave breaking had started just before $t=12 \mathrm{~h}$ in (b). Note that purple indicates wave-induced flow stagnation and reversals.

smoothing operator to Eq. (1) along with these definitions gives

$$
\begin{aligned}
\frac{\partial \tilde{U}}{\partial t}= & -\frac{1}{\langle\bar{\rho}\rangle}\left\langle\frac{\partial\left(\bar{\rho} u^{\prime} w^{\prime}\right)}{\partial z}\right\rangle-\frac{1}{\langle\bar{\rho}\rangle}\left\langle\frac{\partial\left(\bar{\rho} u^{\prime} u^{\prime}\right)}{\partial x}\right\rangle \\
& -\frac{1}{\langle\bar{\rho}\rangle}\left\langle\frac{\partial\langle p\rangle}{\partial x}\right\rangle+\text { OT, }
\end{aligned}
$$

where $\tilde{U}=\langle\bar{\rho} u\rangle /\langle\bar{\rho}\rangle$ is the regional-density-weighted ambient $u$ and OT represents the other terms that result from partitioning. Integrating this budget over time gives an equation for the regional flow deceleration:

$$
\Delta \tilde{U}=\Delta \tilde{U}_{\mathrm{MWD}_{x z}}+\Delta \tilde{U}_{\mathrm{MWD}_{x x}}+\Delta \tilde{U}_{\mathrm{PGF}}+\Delta \tilde{U}_{\mathrm{OT}}
$$

where $\Delta \tilde{U}_{\mathrm{MWD}_{x z}}\left(\Delta \tilde{U}_{\mathrm{MWD}_{x x}}\right)$ is the regional deceleration caused by vertical (horizontal) divergences of vertical (horizontal) momentum flux and $\Delta \tilde{U}_{\mathrm{PGF}}$ is the regional deceleration caused by the pressure gradient force.

The lhs and the first three terms on the rhs of Eq. (28) at $z=50 \mathrm{~km}$ are shown in Fig. 8, integrated over the first (left column) and subsequent (right column) $12 \mathrm{~h}$ of a no-shear case with a $4000-\mathrm{km}$-wide domain and $24 \mathrm{~h}$ of forcing. Similar to the $1000-\mathrm{km}$ domain, wave breaking began just before $t=12 \mathrm{~h}$. The MW forcings $\left(\Delta \tilde{U}_{\mathrm{MWD}_{x z}}, \Delta \tilde{U}_{\mathrm{MWD}_{x x}}\right)$ and responses in the left (right) column of Fig. 8 are then interpreted as nondissipative (dissipative). In both cases, the forcings and responses are qualitatively similar, though larger forcings and responses occurred during wave breaking.

The MW forcings are local, as seen in Figs. 8a, 8b, 8e, and $8 \mathrm{f}$. In the region with MW forcings, there is a complex balance between the forcings and the pressure gradient force (PGF). While the terms plotted here do not close the momentum budget near the mountain (i.e., $|x| \leq 400 \mathrm{~km})$, the budget does nearly close away from the MWs (Figs. 8d,h). The only forcing responsible for decelerations away from the mountain is the PGF. Note also that the flow is not particularly decelerated where MWD is exerted (Figs. 8d,h, black).

We speculate, similar to Durran (1995), that the local forcing is quickly communicated horizontally by a 

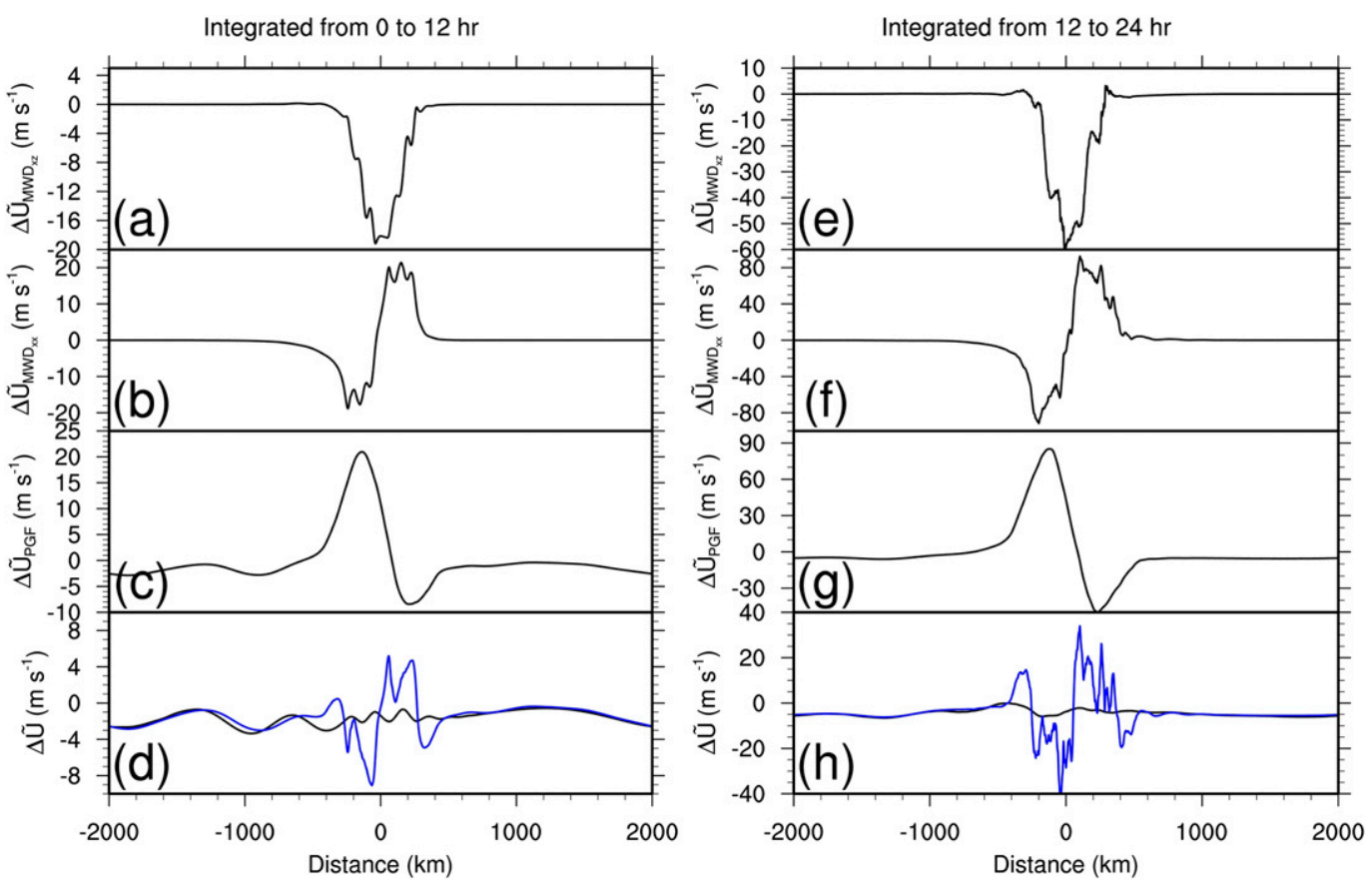

FIG. 8. The contributions of (a),(e) $\Delta \tilde{U}_{\mathrm{MWD}_{x z}}$, (b),(f) $\Delta \tilde{U}_{\mathrm{MWD}_{x x}}$, and (c),(g) $\Delta \tilde{U}_{\mathrm{PGF}}$ to (d),(h) $\Delta \tilde{U}$ in Eq. (28) are shown for the no-shear case with a 4000-km-wide domain. The time integral was computed over the (left) first and (right) subsequent $12 \mathrm{~h}$ of the simulation, corresponding to periods without and with wave breaking, respectively, during the 24-h forcing period. Note the different $y$-axis limits for the different integration periods. The smoothed $\Delta \tilde{U}$ (black) and $\left(\Delta \tilde{U}_{\mathrm{MWD}_{x z}}+\Delta \tilde{U}_{\mathrm{MWD}_{x x}}+\Delta \tilde{U}_{\mathrm{PGF}}\right)$ (blue) are shown in (d) and (h).

combination of fast gravity waves and infrasound waves. These waves set up a broad, adverse PGF away from the mountain (Figs. 8c,g) that uniformly slows the flow. This result justifies the domain-averaged analysis primarily presented in this study.

\section{c. Nondissipative and dissipative interactions}

The MF evolution for the breaking MW cases is shown in Figs. 9a-c and 9d-f for the WRF and FR solutions, respectively. In all three shear cases, there is good agreement between the WRF and FR solutions prior to wave breaking, which initiates near $t \approx 11.67,9$, and $8 \mathrm{~h}$ in the zero-, positive-, and negative-shear cases, respectively. After wave breaking begins, dissipative MF reductions relative to the nondissipative FR predictions are apparent, especially in the zero- and positive-shear cases.

The ambient decelerations in the WRF and FR solutions are shown in Figs. 10a-1. Prior to wave breaking, nondissipative decelerations occur in the WRF solutions, which agree qualitatively with the FR solutions. These $\Delta U_{\text {nd }}$ are substantial. In the no-shear and positive-shear cases, $\Delta U_{\text {nd }}$ decelerate the initial profile by $\sim 30 \%$ prior to the onset of wave breaking, which likely influence the MW's evolution to breakdown. However, once wave breaking initiates, dissipative decelerations quickly develop, increase, and dominate the total decelerations, especially in the no-shear and positive-shear cases. At the end of the simulations, only dissipative decelerations remain.

After wave breaking initiates, permanent $\Delta U_{d}$ develops in layers, evident as horizontal streaks (Figs. 10a-c, g-i). Similar to the small-mountain case in section 5, this layering appears to be initiated by enhanced momentum deposition in the vertically recurring and localized regions of steepening above the highest terrain by hydrostatic MWs forced by horizontally localized terrain (e.g., Figs. 7b,e; Smith 1977). The layers with enhanced momentum deposition decelerate, subsequently enhancing MWD and $\Delta U_{d}$. While the vertical spacing of the $\Delta U_{d}$ layers does not exactly match $\lambda_{z}=2 \pi U / N$ in the tall-mountain case, the layer spacing does appear proportional to $U$, consistent with this isolated mountain steepening mechanism.

\section{Influence of event duration}

As hinted in Fig. 5, the completeness of the MW spectrum aloft is dependent upon the event duration, which has implications for MW evolution and dissipation. In this section, the influence of event duration is investigated in WRF simulations with durations from $\tau=1$ to $36 \mathrm{~h}$ in the three shear cases. 


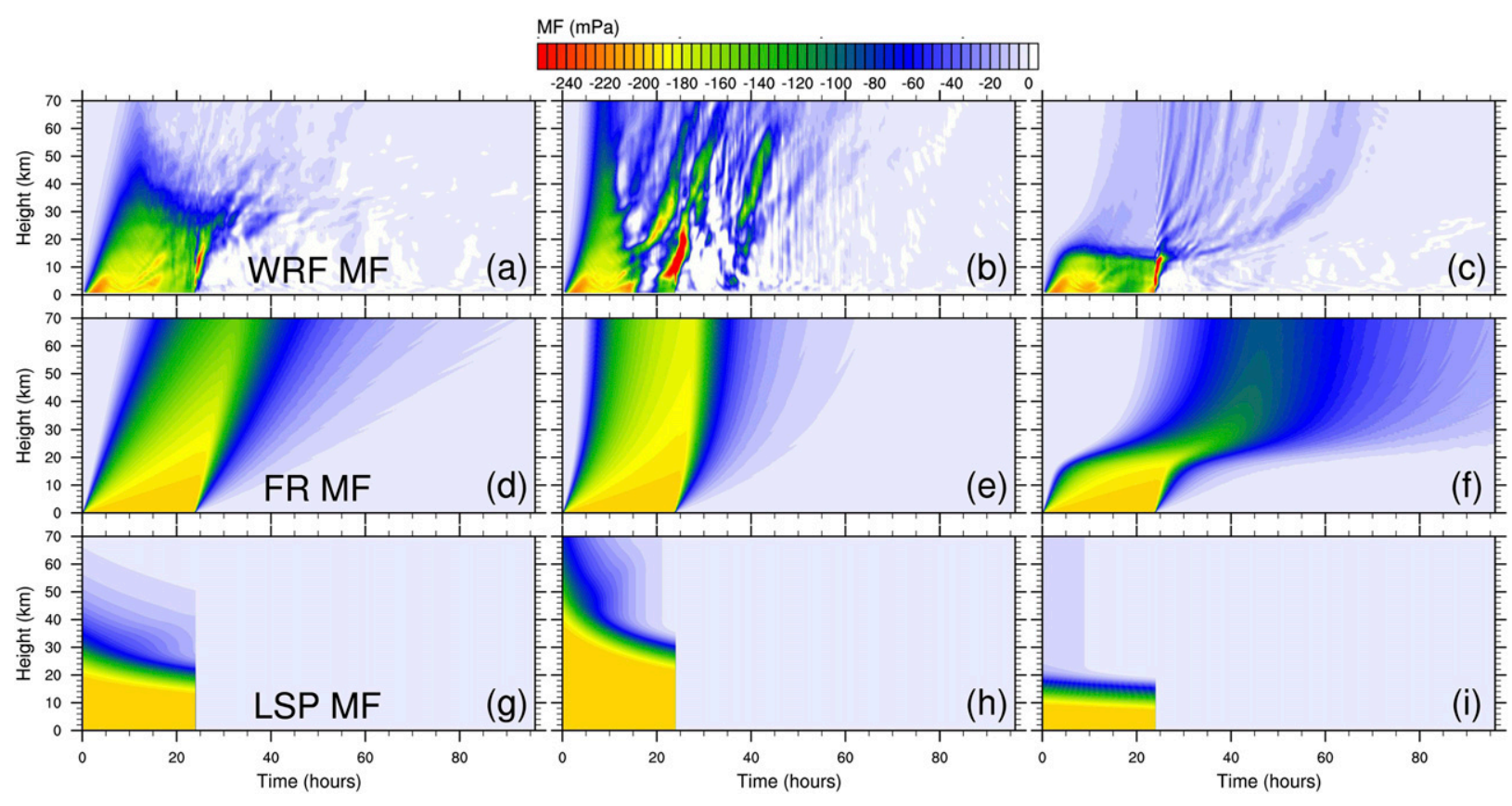

FIG. 9. Horizontal domain-averaged vertical flux of zonal momentum (MF $=\bar{\rho} \overline{u^{\prime} w^{\prime}}, \mathrm{mPa}$ ) as a function of height and time from the (a)-(c) WRF, (d)-(f) FR, and (g)-(i) LSP models for the tall mountain $\left(h_{m}=500 \mathrm{~m}\right)$. Results are from the (left) no-shear, (center) positive-shear, and (right) negative-shear cases.

The final $(t=96 \mathrm{~h})$ total $\Delta U$ in $36 \mathrm{WRF}$ solutions with event durations of $\tau=1,2, \ldots, 36 \mathrm{~h}$ are plotted as a function of event duration in Fig. 11 for the zero-shear (Fig. 11a), positive-shear (Fig. 11b), and negative-shear (Fig. 11c) cases. By $t=96 \mathrm{~h}$, at least $60 \mathrm{~h}$ after the forcing, these decelerations are interpreted as entirely dissipative. In all shear cases, the lowest breaking level $z_{b}$ (i.e., lowest $\left.\Delta U_{d}<0\right)$ decreases with increasing event duration consistent with Lott and Tietelbaum (1993). This dependence is especially sensitive for shorter events $(\tau \lesssim 15 \mathrm{~h})$.

Because of the $c_{g z}$ spectrum (i.e., vertical dispersion), the completeness of the MW spectrum aloft depends on both event duration and altitude. As demonstrated in Fig. 5, longer event durations allow a more complete spectrum, as the slow long-wave portion of the spectrum has more time to propagate up prior to the end of the forcing and departure of the fast short waves. At lower altitudes, less time is required by all wave components to propagate to the level considered, allowing a more complete spectrum as well. MW amplitude (e.g., $u^{\prime}$ variance) increases with spectrum completeness. Hence, forcing duration and altitude influence the maximum MW amplitude, neglecting the influence of density.

For short events, spectrum completeness controls MW amplitude and $z_{b}$. In order for MWs to break, they must propagate many density scale heights (e.g., Fig. 11a) to overcome their limited amplitude caused by limited spectrum completeness. This effect, along with the decrease in nonlinearity with increasing wind, results in no wave breaking in the positive-shear case for $\tau \lesssim 5 \mathrm{~h}$. As event duration and MW amplitude increases, $z_{b}$ decreases. The wind reduction in the negative-shear case, which essentially forces nonlinearity and wave breaking, occurred at a relatively low altitude, so event duration has less of an influence on $z_{b}$.

For longer events, descending negative-shear-level dynamics might influence $z_{b}$ as well, allowed by the periodic lateral boundary conditions. As MWs dissipatively decelerate the ambient flow, a layer of negative shear (relative to the initial profile) develops below. Subsequent MWD might occur in the negative shear below the previous MWD, lowering this negative-shear layer and flow decelerations with time while MW forcing continues. This feature is not apparent in the no-shear or positive-shear cases (Figs. 11a,b). Layers of decelerated flow are further decelerated, even when the flow is reversed, rather than lowering and spreading with time when the forcing duration is increased. Note that the WRF $z_{b}$ closely follows the FR predictions of the lowest overturning level (black line in Figs. 11a-c) in the zeroand positive-shear cases. This indicates that spectrum completeness determines $z_{b}$ and not the descending negative-shear-level mechanism. The negative-shear case is the exception, where the WRF $z_{b}$ descends below the FR predictions with time, suggesting the descending shear-level mechanism is active in this case. 


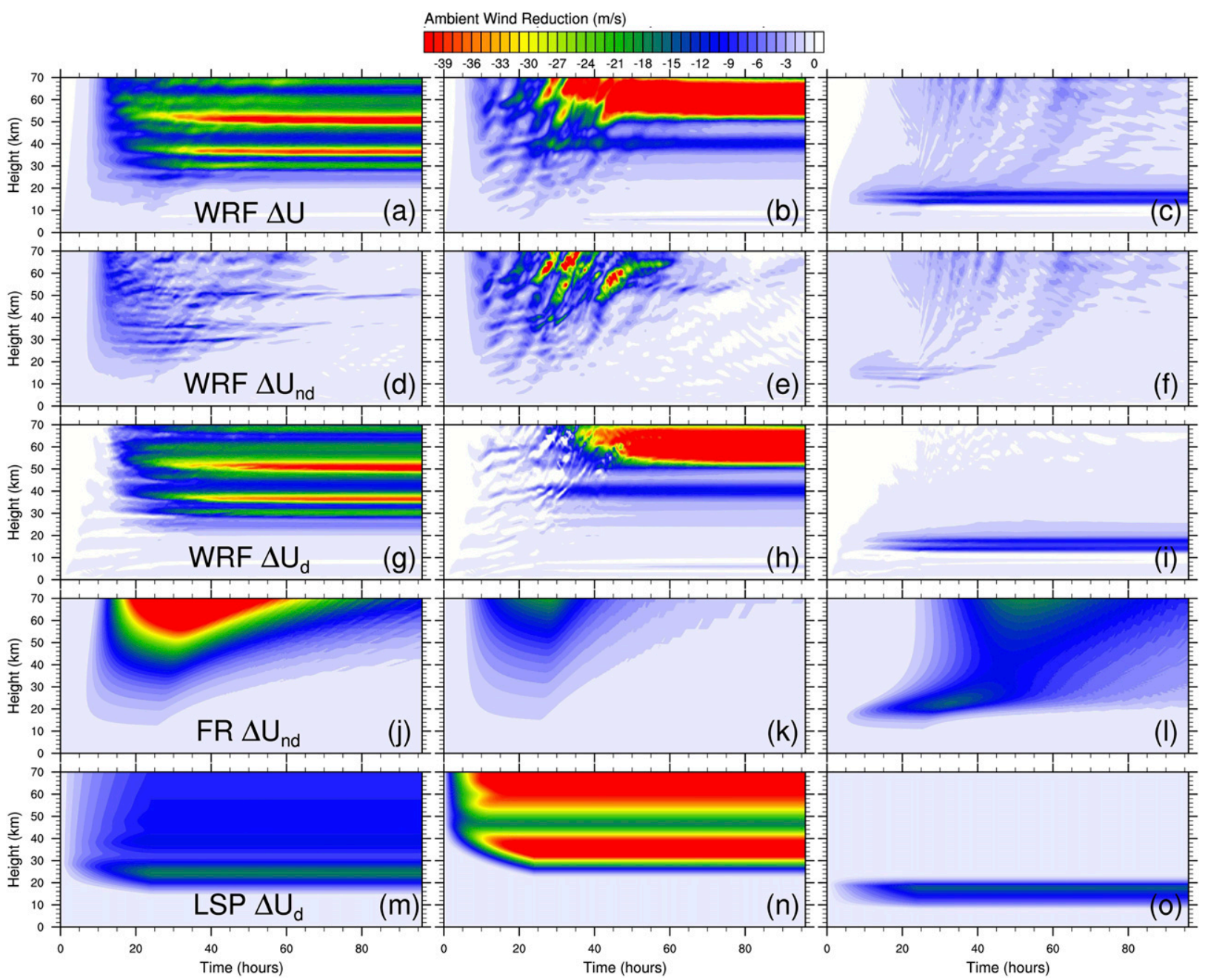

FIG. 10. Nondissipative and dissipative ambient flow decelerations $\left(\mathrm{m} \mathrm{s}^{-1}\right)$ are shown as a function of height and time from the WRF (top three rows), FR (fourth row), and LSP (bottom row) models for the tall mountain $\left(h_{m}=500 \mathrm{~m}\right)$. The total decelerations $\Delta U$, nondissipative decelerations $\Delta U_{\text {nd }}$ computed via Eq. (6), and dissipative decelerations $\left(\Delta U_{d}=\Delta U-\Delta U_{\text {nd }}\right)$ are shown in the WRF solutions in (a)-(c), (d)-(f), and (g)-(i), respectively. Results are from the (left) no-shear, (center) positive-shear, and (right) negativeshear cases.

Note that the FR model predicts stepped decreases in $z_{b}$ with increasing duration, and that these steps roughly predict the altitude and appearance of the $\Delta U_{d}$ layers in the zero- and positive-shear WRF solutions (Figs. 11a,b). This further supports the idea that the $\Delta U_{d}$ layering is due to enhanced MWD in the localized overturning regions of MWs forced by localized terrain.

\section{Parameterization evaluation}

Here, the instantaneous and monochromatic LSP model (described in section $4 \mathrm{c}$ ) is quantitatively compared against the transient, broad-spectrum WRF solutions. The comparison is of $\Delta U_{d}$ structures, the lowest breaking levels $z_{b}$, and the total impulse by MWD $J_{\text {MWD. }}$.

In the LSP solutions, wave breaking initiates immediately by design (Figs. 9g-i), immediately decelerating the flow aloft (Figs. 10m-o). Negative shear (relative to the initial profiles) develops below the initial $z_{b}$ (Table 1), allowing enhanced subsequent MW dissipation at and below these levels. That is, the descending negativeshear-level mechanism results in descent of the levels of dissipation and deceleration with time, explaining the salient feature in Figs. 9g-i and 10m-o. Additionally, almost all of the MW dissipation takes place in this negative shear once it develops. The rate of descent is dependent on the density at $z_{b}$, leading to the strongest decelerations and the fastest descent in the positive-shear case. 


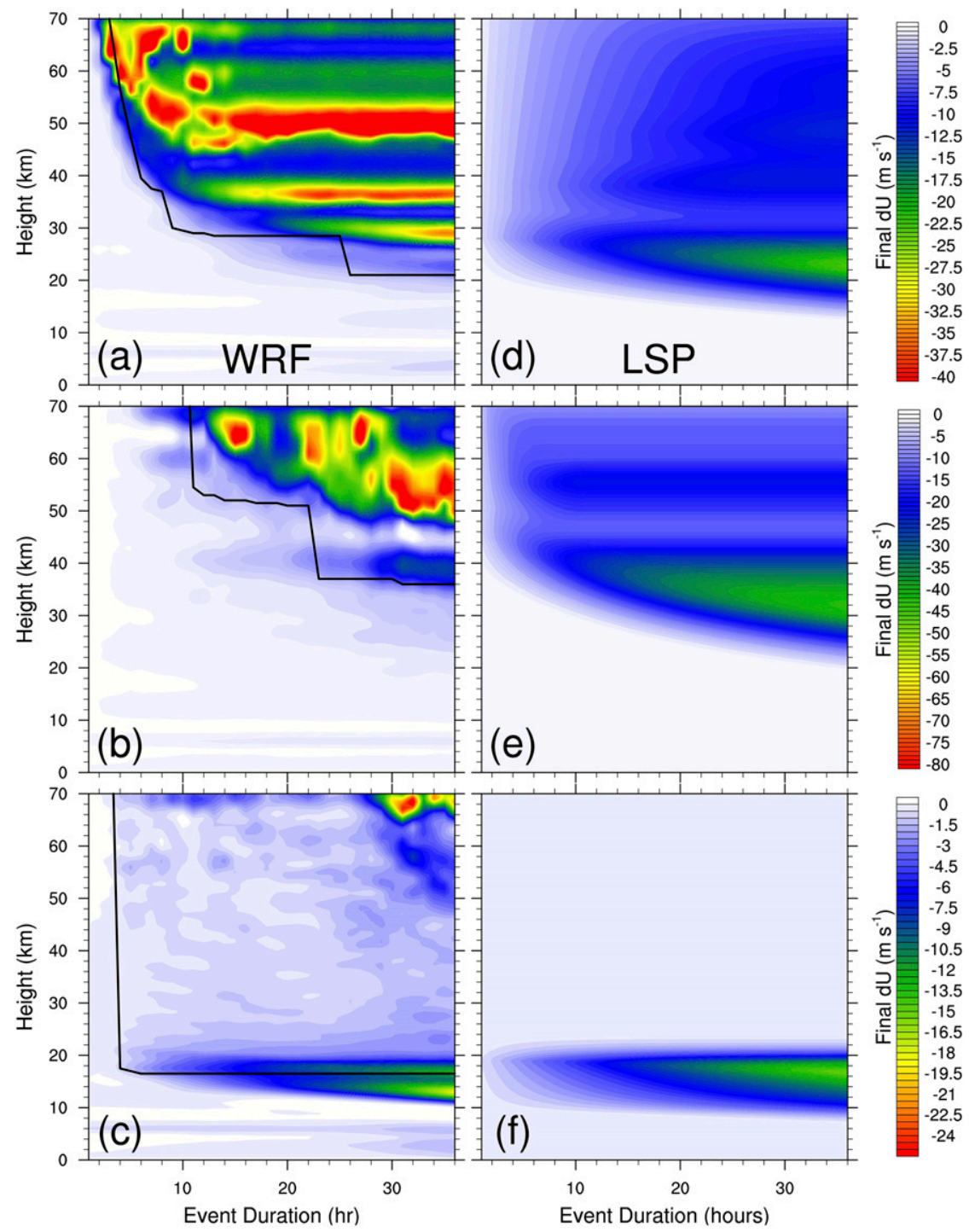

FIG. 11. Final $(t=96 \mathrm{~h}) \Delta U\left(\mathrm{~m} \mathrm{~s}^{-1}\right)$ as a function of forcing duration and height from the (a)-(c) WRF and (d)-(f) LSP solutions. Results are from the (a),(d) no-shear, (b),(e) positiveshear, and (c),(f) negative-shear cases. The black solid line in (a)-(c) depicts the lowest breaking level predicted by the FR model.

Final $(t=96 \mathrm{~h}) \Delta U_{d}$ in the LSP solutions are plotted as a function of duration and compared with WRF solutions in Fig. 11. Similar to Fig. 10, qualitatively, the LSP model performs best in the negative-shear case and performs quite poorly in the no-shear and positive-shear cases. In all cases, the LSP model obviously fails to produce the $\Delta U_{d}$ layering in the WRF solutions. Much of the disagreement between the two solutions (other than the $\Delta U_{d}$ layering) is due to the instant propagation and monochromatic assumptions in the LSP model, which result in incorrect timing and levels of wave breaking and too much momentum deposition.
Consider a situation where only the monochromatic assumption is relaxed. The full spectrum would be present at all altitudes instantly, allowing the full wave amplitude and wave breaking to occur instantly. Wave breaking occurs much lower than in a transient, fullspectrum case, especially for events with short forcing duration (cf. Table 1; Figs. 11a-c). Wave breaking occurs too early as well. In the positive-shear case, vertical dispersion and increasing winds prevent breaking from occurring at all below $70 \mathrm{~km}$ for durations $\leq 10 \mathrm{~h}$, whereas instant propagation would result in breaking as low as $35.5 \mathrm{~km}$. 
TABLE 1. Minimum breaking levels $z_{b}(\mathrm{~km})$ using the instant propagation assumption in monochromatic and full-spectrum calculations. In the monochromatic case, this is the initial $z_{b}$ in the LSP solutions. The full-spectrum $z_{b}$ is the lowest overturning altitude in a solution by the FR model in the steady $\left(F_{\mathrm{sfc}}=1\right)$ configuration. In both cases, the pressure drag was $\mathrm{MF}_{0}=196.504 \mathrm{mPa}$ over a 1000-km-wide domain. Transient, full-spectrum FR predictions of $z_{b}$ are shown as a function of forcing duration in Figs. 11a-c.

\begin{tabular}{lcc}
\hline \hline \multicolumn{1}{c}{$z_{b}$} & $\lambda_{x}=328(\mathrm{~km})$ & Full spectrum $(\mathrm{km})$ \\
\hline No shear & 24.0 & 20.5 \\
Positive shear & 41.5 & 35.5 \\
Negative shear & 14.5 & 16.5 \\
\hline
\end{tabular}

Similar errors result for an instant, monochromatic wave with the broad-spectrum MF assigned to it (cf. Table 1; Fig. 11). Here, there is the added complication that $z_{b}$ depends on the parameterized wavelength. For example, in the no-shear case, assuming density decreases with a single scale height $H_{\rho}, z_{b}=H_{\rho} \ln \left[\bar{\rho}_{0} U_{0}^{3} k_{p} /\left(2 N \mathrm{MF}_{0}\right)\right]$ is increases with increasing $k_{p}$. The parameterized wavelength might be tuned to produce correct $z_{b}$ for a particular terrain in the steady case, but this tuning will not be correct in general for broad-spectrum complex terrain and certainly not correct for transient, broad-spectrum MWs.

Now consider a monochromatic, transient parameterized MW in a steady ambient flow. The level and duration of breaking would be unaffected (neglecting $\Delta U_{\mathrm{nd}}$ ), though the timing would be shifted by the time to propagate. After the event, $\Delta U_{d}$ would be the same as in the instant and monochromatic cases. Hence, both transient and full-spectrum dynamics must be considered to accurately predict the levels and timing $z_{b}$.

These assumptions influence how much momentum deposition occurs below some height as well. The $x$-impulse budget [Eq. (11); $J_{0}=J_{\mathrm{MWD}}+J_{z_{d}}$ ] is reported in Table 2. Here, the $\mathrm{MF}_{0}$ in the WRF solutions was supplied to the LSP model so that $J_{0}$ was identical in both solutions. This allows the MWD impulse $\left(J_{\mathrm{MWD}}\right)$ exerted below and mountain impulse transmitted through $z_{d}=70 \mathrm{~km}\left(J_{z_{d}}\right)$ to be directly compared. While the choice of $z_{d}$ is somewhat arbitrary, it roughly represents the typical model top height of current global weather prediction and climate models. In all cases, the "optimally tuned" (i.e., given correct source drag, wavenumber of peak in the MF spectrum) LSP model deposits too much momentum below $z_{d}$ (i.e., $\left|J_{\text {MWD }}\right|$ larger in LSP solutions). The overestimation is largest in the positive-shear case, where the LSP error relative to the WRF solution is $\sim 50 \%-100 \%$.

The amount of $J_{0}$ exerted below $z_{d}\left(J_{\mathrm{MWD}}\right)$ is limited by the escape of MWs through $z_{d}\left(J_{z_{d}}\right)$. In both the WRF and LSP solutions, this escape happens early (cf. Figs. 9a-c and $9 \mathrm{~g}-\mathrm{i})$, prior to the development of strong decelerations and nearly complete MW dissipation. In the LSP solutions, wave breaking occurs instantly and the initial escape is only due to incomplete dissipation allowed by the saturation assumption. However, in the WRF solutions, some MF escapes prior to wave breaking. This is possible because the initial spectrum is quite incomplete, with only the short, fast MWs present aloft, and because these short MWs carry MF but contribute little to $u^{\prime}$ variance (Smith and Kruse 2017). Both influences inhibit wave amplitudes and allow transmission through $z_{d}$ without overturning. Eventually, longer waves with larger contributions to $u^{\prime}$ variance build in and the MWs overturn at increasingly lower altitudes. Some escape occurs because of incomplete dissipation as well, but the escape of the fast, short waves prior to breaking is the primary reason why the MWD impulse is less in the WRF solutions.

Qualitatively, the best agreement between the LSP and WRF solutions occurred in the negative-shear case (Figs. 10, 11), consistent with the comparison between full-physics, realistic WRF simulations against MERRAand MERRA2-parameterized MWD in the lowerstratospheric MW "valve layer" (Kruse et al. 2016). This is due to both the increase in nonlinearity by negative shear and the low altitude of this shear. The weak winds increase nonlinearity (i.e., $\hat{u} / U \propto U^{-3 / 2}$ ), making $z_{b}$ less sensitive to the choice of $k_{p}$ or spectrum completeness (cf. Table 1; Fig. 11). Also, the instant-propagation assumption is best met in this case because of the relatively low altitude of the negative shear. However, the optimally tuned LSP model again overestimates MW momentum deposition, as it did in all cases (Table 2).

\section{Summary and conclusions}

The question posed by this paper is how transient, broad-spectrum wave dynamics influence MW evolution and momentum deposition from generation to dissipation. By introducing transience, MWs can influence their ambient flow nondissipatively as they propagate into and out of layers aloft, in addition to dissipatively. A broad spectrum of MWs introduces a $c_{g z}$ spectrum, which vertically disperses MWs and influences spectrum completeness. Spectrum completeness, in turn, influences MW amplitude (e.g., $\left.u^{\prime}\right), \mathrm{MF}$, and nondissipative decelerations $\Delta U_{\text {nd }}$.

Both altitude and event duration influence spectrum completeness. During the forcing period at some level aloft, fast, short waves arrive first, with longer waves arriving with time, increasing spectrum completeness. After the forcing period, the fast, short waves are the first to depart. A short-wave portion of the spectrum departs prior to the arrival of a slow, long portion of the spectrum, limiting spectrum completeness. As time increases after the forcing, the MW spectrum becomes increasingly narrow and shifts to longer scales. The $c_{g z}$ 
TABLE 2. The impulse budget terms $\left(\mathrm{GN} \mathrm{s} \mathrm{m}^{-1}\right)$ in Eq. (11) in the WRF and LSP solutions with forcing durations of 12 and $24 \mathrm{~h}$. All impulses were computed from integrals over the entire 96-h simulations. Here, the forcing-duration-averaged pressure drag in WRF was supplied to the LSP model to ensure the same mountain impulse $\left(J_{0}\right)$, allowing a direct comparison between the MWD impulse exerted below $\left(J_{\mathrm{MWD}}\right)$ and the impulse transmitted through $z_{d}=70 \mathrm{~km}\left(J_{z_{d}}\right)$. LSP errors in $J_{\mathrm{MWD}}$ relative to the WRF solutions [i.e., (LSP - WRF)/LSP] are given in the last row.

\begin{tabular}{|c|c|c|c|c|c|c|c|c|c|c|c|c|}
\hline \multirow{3}{*}{$\begin{array}{c}\text { Budget } \\
\text { term }\end{array}$} & \multicolumn{6}{|c|}{$\tau=12 \mathrm{~h}$} & \multicolumn{6}{|c|}{$\tau=24 \mathrm{~h}$} \\
\hline & \multicolumn{2}{|c|}{ Zero shear } & \multicolumn{2}{|c|}{ Positive shear } & \multicolumn{2}{|c|}{ Negative shear } & \multicolumn{2}{|c|}{ Zero shear } & \multicolumn{2}{|c|}{ Positive shear } & \multicolumn{2}{|c|}{ Negative shear } \\
\hline & WRF & LSP & WRF & LSP & WRF & LSP & WRF & LSP & WRF & LSP & WRF & LSP \\
\hline$J_{0}$ & \multicolumn{2}{|c|}{-8.34} & \multicolumn{2}{|c|}{-8.40} & \multicolumn{2}{|c|}{-8.49} & \multicolumn{2}{|c|}{-16.17} & \multicolumn{2}{|c|}{-13.88} & \multicolumn{2}{|c|}{-15.71} \\
\hline$J_{\mathrm{MWD}}$ & -7.09 & -8.22 & -2.94 & -6.31 & -6.11 & -8.20 & -15.12 & -15.95 & -7.54 & -11.63 & -13.42 & -15.37 \\
\hline$J_{z_{d}}$ & -1.34 & -0.13 & -5.61 & -2.09 & -2.56 & -0.30 & -1.33 & -0.22 & -6.58 & -2.24 & -2.55 & -0.35 \\
\hline$J_{\mathrm{MWD}}$ error $(\%)$ & \multicolumn{2}{|c|}{15.9} & \multicolumn{2}{|c|}{114.6} & \multicolumn{2}{|c|}{34.2} & \multicolumn{2}{|c|}{5.5} & \multicolumn{2}{|c|}{54.2} & \multicolumn{2}{|c|}{14.5} \\
\hline
\end{tabular}

spectrum has less of an impact on spectrum completeness at lower altitudes.

The shape of the source spectrum, along with its completeness, determines wave amplitude (e.g., $u^{\prime}$ variance), MF, and $\Delta U_{\text {nd }}$. The $\Delta U_{\text {nd }}$ spectrum [Eq. (10)] has the same shape as the $u^{\prime}$ variance spectrum (multiplied by a negative constant), which is dominated by long waves (Smith and Kruse 2017). While MW spectra become increasingly narrow after a forcing period, they are shifted to longer scales with larger contributions to $\Delta U_{\text {nd }}$ and $u^{\prime}$ variance. Because of the slow vertical propagation of these longer scales, it can take days for nondissipating MWs to depart the middle atmosphere and allow the ambient flow to recover, especially in cases with weak winds in the middle atmosphere.

In cases with MW breaking, $\Delta U_{\text {nd }}$ can be substantial. For example, in the zero- and negative-shear cases studied here, nondissipative MWD decelerates the ambient flow by up to $\sim 30 \%$ prior to wave breaking. These nondissipative decelerations are nonnegligible and influence the evolution of MWs to breaking. However, breaking of the short, fast MWs quickly initiates, which reduces nondissipative decelerations (i.e., pseudomomentum) and causes dissipative decelerations to develop. MW breaking continues as slower, longer MWs arrive and break, allowing dissipative decelerations to continue to accumulate and dominate the total decelerations.

Forcing duration influences the lowest breaking level of an MW event through its influence on spectrum completeness and wave amplitude. Spectrum completeness determines both the initial and (at least in zero- and positive-shear environments) the subsequent lowest breaking levels during a single event. As forcing duration increases, spectrum completeness and wave amplitudes increase, which decrease the altitude at which waves can overturn in an MW event.

These results are relevant to the MWD parameterization problem, as these transient, broad-spectrum dynamics are neglected by the conventional instant propagation and monochromatic assumptions. These two assumptions result in MW breaking occurring too early (i.e., instantly) and too low. Additionally, these assumptions prevent the transmission of fast, short MWs prior to breaking, leading to an overestimation of MWD impulse. The largest parameterization errors occurred in the case with positive ambient wind shear, where dissipative MW impulse deposited below $z=$ $70 \mathrm{~km}$ was $\sim 50 \%-100 \%$ too high. Qualitatively, the parameterization performed the best in the negative-shear case. These results suggest that "optimally tuned" (i.e., having the correct source drag, the most appropriate wavelength) instant, monochromatic MWD parameterizations may perform the best in winter midlatitudes, where winds are reduced in the lower stratosphere but have the largest errors at latitudes below the stratospheric polar night jet, where wind shear is broadly positive to $z=50 \mathrm{~km}$. The results presented here motivate the development of a transient, broad-spectrum MWD parameterization, which could improve timing and levels of wave breaking and apply a more accurate total amount of MWD in weather and climate models.

Acknowledgments. This work was supported by the National Science Foundation (NSF-AGS-1338655). High-performance computing was performed on the Yellowstone supercomputer (ark:/85065/d7wd3xhc) with support provided by NCAR's Computational and Information Systems Laboratory, sponsored by the National Science Foundation. We would also like to acknowledge the useful discussions with Julio Bacmeister during the preparation of this manuscript and the very helpful comments and critiques by Oliver Bühler, Ulrich Achatz, and one anonymous reviewer.

\section{REFERENCES}

Achatz, U., R. Klein, and F. Senf, 2010: Gravity waves, scale asymptotics and the pseudo-incompressible equations. J. Fluid Mech., 663, 120-147, https://doi.org/10.1017/S0022112010003411.

Alexander, M. J., and Coauthors, 2010: Recent developments in gravity-wave effects in climate models and the global distribution 
of gravity-wave momentum flux from observations and models. Quart. J. Roy. Meteor. Soc., 136, 1103-1124, https://doi.org/10.1002/ qj.637.

Andrews, D. G., and M. E. Mcintyre, 1978: An exact theory of nonlinear waves on a Lagrangian-mean flow. J. Fluid Mech., 89, 609-646, https://doi.org/10.1017/S0022112078002773.

Bölöni, G., B. Ribstein, J. Muraschko, C. Sgoff, J. Wei, and U. Achatz, 2016: The interaction between atmospheric gravity waves and large-scale flows: An efficient description beyond the nonacceleration paradigm. J. Atmos. Sci., 73, 4833-4852, https://doi.org/10.1175/JAS-D-16-0069.1.

Bretherton, F. P., 1969: Momentum transport by gravity waves. Quart. J. Roy. Meteor. Soc., 95, 213-243, https://doi.org/ 10.1002/qj.49709540402.

Broutman, D., J. W. Rottman, and S. D. Eckermann, 2002: Maslov's method for stationary hydrostatic mountain waves. Quart. J. Roy. Meteor. Soc., 128, 1159-1171, https://doi.org/10.1256/ 003590002320373247.

_ _ J. Ma, S. D. Eckermann, and J. Lindeman, 2006: Fourier-ray modeling of transient trapped lee waves. Mon. Wea. Rev., 134, 2849-2856, https://doi.org/10.1175/MWR3232.1.

Bühler, O., 2009: Waves and Mean Flows. Cambridge University Press, $341 \mathrm{pp}$

— and M. E. McIntyre, 2003: Remote recoil: A new wave-mean interaction effect. J. Fluid Mech., 492, 207-230, https://doi.org/ 10.1017/S0022112003005639.

— and - 2005: Wave capture and wave-vortex duality. J. Fluid Mech., 534, 67-95, https://doi.org/10.1017/S0022112005004374.

Chen, C.-C., D. R. Durran, and G. J. Hakim, 2005: Mountain-wave momentum flux in an evolving synoptic-scale flow. J. Atmos. Sci., 62, 3213-3231, https://doi.org/10.1175/JAS3543.1.

_ , G. J. Hakim, and D. R. Durran, 2007: Transient mountain waves and their interaction with large scales. J. Atmos. Sci., 64, 2378-2400, https://doi.org/10.1175/JAS3972.1.

Dosser, H., and B. Sutherland, 2011: Weakly nonlinear nonBoussinesq internal gravity wavepackets. Physica D, 240, 346-356, https://doi.org/10.1016/j.physd.2010.09.008.

Durran, D. R., 1995: Do breaking mountain waves deceierate the local mean flow? J. Atmos. Sci., 52, 4010-4032, https://doi.org/ 10.1175/1520-0469(1995)052<4010:DBMWDT>2.0.CO;2.

Eckermann, S. D., J. Ma, and D. Broutman, 2015: Effects of horizontal geometrical spreading on the parameterization of orographic gravity wave drag. Part I: Numerical transform solutions. J. Atmos. Sci., 72, 2330-2347, https://doi.org/10.1175/JAS-D-14-0147.1.

_ - and Coauthors, 2016: Dynamics of orographic gravity waves observed in the mesosphere over the Auckland Islands during the Deep Propagating Gravity Wave Experiment (DEEPWAVE). J. Atmos. Sci., 73, 3855-3876, https://doi.org/ 10.1175/JAS-D-16-0059.1.

Eliassen, A., and E. Palm, 1960: On the transfer of energy in stationary mountain waves. Geofys. Publ., 22 (3), 1-23.

Fritts, D. C., and T. J. Dunkerton, 1984: A quasi-linear study of gravity-wave saturation and self-acceleration. J. Atmos. Sci., 41, 3272-3289, https://doi.org/10.1175/1520-0469(1984) $041<3272$ :AQLSOG $>2.0$.CO;2.

- , B. Laughman, T. S. Lund, and J. B. Snively, 2015: Self-acceleration and instability of gravity wave packets: 1 . Effects of temporal localization. J. Geophys. Res. Atmos., 120, 8783 8803, https://doi.org/10.1002/2015JD023363.

Garcia, R. R., and S. Solomon, 1985: The effect of breaking gravity waves on the dynamics and chemical composition of the mesosphere and lower thermosphere. J. Geophys. Res., 90, 3850 3868, https://doi.org/10.1029/JD090iD02p03850.
Gregory, D., G. J. Shutts, and J. R. Mitchell, 1998: A new gravitywave-drag scheme incorporating anisotropic orography and low-level wave breaking: Impact upon the climate of the UK Meteorological Office Unified Model. Quart. J. Roy. Meteor. Soc., 124, 463-493, https://doi.org/10.1002/qj.49712454606.

Hasha, A., O. Bühler, and J. Scinocca, 2008: Gravity wave refraction by three-dimensionally varying winds and the global transport of angular momentum. J. Atmos. Sci., 65, 2892-2906, https://doi.org/10.1175/2007JAS2561.1.

Holton, J. R., 1983: The influence of gravity wave breaking on the general circulation of the middle atmosphere. J. Atmos. Sci., 40, 2497-2507, https://doi.org/10.1175/1520-0469(1983)040<2497: TIOGWB $>2.0 . \mathrm{CO} ; 2$.

Kim, Y.-J., and A. Arakawa, 1995: Improvement of orographic gravity wave parameterization using a mesoscale gravity wave model. J. Atmos. Sci., 52, 1875-1902, https://doi.org/10.1175/ 1520-0469(1995)052<1875:IOOGWP $>2.0$.CO;2.

- and J. D. Doyle, 2005: Extension of an orographic-drag parametrization scheme to incorporate orographic anisotropy and flow blocking. Quart. J. Roy. Meteor. Soc., 131, 1893-1921, https://doi.org/10.1256/qj.04.160.

Kruse, C. G., R. B. Smith, and S. D. Eckermann, 2016: The midlatitude lower-stratospheric mountain wave valve layer. J. Atmos. Sci., 73, 5081-5100, https://doi.org/10.1175/JAS-D-16-0173.1.

Lindzen, R. S., 1981: Turbulence and stress owing to gravity wave and tidal breakdown. J. Geophys. Res., 86, 9707-9714, https:// doi.org/10.1029/JC086iC10p09707.

Lott, F., and H. Tietelbaum, 1993: Linear unsteady mountain waves. Tellus, 45, 201-220, https://doi.org/10.3402/tellusa.v45i3.14871. and M. J. Miller, 1997: A new subgrid-scale orographic drag parametrization: Its formulation and testing. Quart. J. Roy. Meteor. Soc., 123, 101-127, https://doi.org/10.1002/qj.49712353704.

McFarlane, N. A., 1987: The effect of orographically excited gravity wave drag on the general circulation of the lower stratosphere and troposphere. J. Atmos. Sci., 44, 1775-1800, https://doi.org/ 10.1175/1520-0469(1987)044<1775:TEOOEG > 2.0.CO;2.

McLandress, C., 1998: On the importance of gravity waves in the middle atmosphere and their parameterization in general circulation models. J. Atmos. Sol.-Terr. Phys., 60, 1357-1383, https://doi.org/10.1016/S1364-6826(98)00061-3.

Miles, J. W., 1969: Waves and wave drag in stratified flows. Applied Mechanics: Proceedings of the Twelfth International Congress of Applied Mechanics, M. Hetényi and W. G. Vincenti, Eds, IUTAM Series, Springer, 50-75, https://doi.org/10.1007/978-3-642-85640-2_4.

Miller, M. J., T. N. Palmer, and R. Swinbank, 1989: Parameterization and influence of subgridscale orography in general circulation model and weather prediction models. Meteor. Atmos. Phys., 40, 84-209, https://doi.org/10.1007/BF01027469.

Palmer, T. N., G. J. Shutts, and R. Swinbank, 1986: Alleviation of a systematic westerly bias in general circulation and numerical weather prediction models through an orographic gravity wave drag parametrization. Quart. J. Roy. Meteor. Soc., 112, 1001-1039, https://doi.org/10.1002/qj.49711247406.

Portele, T. C., A. Dörnbrack, J. S. Wagner, S. Gisinger, B. Ehard, P.-D. Pautet, and M. Rapp, 2018: Mountain-wave propagation under transient tropospheric forcing: A DEEPWAVE case study. Mon. Wea. Rev., 146, 1861-1888, https://doi.org/ 10.1175/MWR-D-17-0080.1.

Rieper, F., U. Achatz, and R. Klein, 2013: Range of validity of an extended WKB theory for atmospheric gravity waves: Onedimensional and two-dimensional case. J. Fluid Mech., 729, 330-363, https://doi.org/10.1017/jfm.2013.307. 
Scinocca, J. F., and T. G. Shepherd, 1992: Nonlinear wave-activity conservation laws and Hamiltonian structure for the two-dimensional anelastic equations. J. Atmos. Sci., 49, 5-28, https://doi.org/10.1175/ 1520-0469(1992)049<0005:NWACLA > 2.0.CO;2.

Skamarock, W. C., and Coauthors, 2008: A description of the Advanced Research WRF version 3. NCAR Tech. Note NCAR/TN-475+STR, 113 pp., http://dx.doi.org/10.5065/ D68S4MVH.

Smith, R. B., 1977: The steepening of hydrostatic mountain waves. J. Atmos. Sci., 34, 1634-1654, https://doi.org/10.1175/15200469(1977)034<1634:TSOHMW>2.0.CO;2.

_ 1979: The influence of mountains on the atmosphere. $A d$ vances in Geophysics, B. Saltzman, Ed., Vol. 21, Academic Press, 87-230, https://doi.org/10.1016/S0065-2687(08)60262-9.
— J. Atmos. Sci., 74, 1381-1402, https://doi.org/10.1175/JAS-D16-0297.1.

$\longrightarrow$, and - 2018: A gravity wave drag matrix for complex terrain. J. Atmos. Sci., https://doi.org/10.1175/JAS-D-17-0380.1, in press.

van den Bremer, T. S., and B. R. Sutherland, 2014: The mean flow and long waves induced by two-dimensional internal gravity wavepackets. Phys. Fluids, 26, 106601, https://doi.org/10.1063/ 1.4899262.

Webster, S., A. R. Brown, D. R. Cameron, and C. P. Jones, 2003: Improvements to the representation of orography in the Met Office Unified Model. Quart. J. Roy. Meteor. Soc., 129, 19892010, https://doi.org/10.1256/qj.02.133. 